\title{
Artemin is Upregulated by TrkB agonist and Protects the Immature Retina against Hypoxic- ischemic Injury by Suppressing Neuroinflammation and Astrogliosis
}

\section{Hsiu-Mei Huang}

Chang Gung Memorial Hospital Kaohsiung Branch

Chao-Ching Huang

National Cheng Kung University Hospital

\section{Linda Yi-Chieh Poon}

Chang Gung Memorial Hospital Kaohsiung Branch

Ying-Chao Chang ( $\nabla$ ycchao@cgmh.org.tw )

Chang Gung Memorial Hospital Kaohsiung Branch

\section{Research}

Keywords: Artemin (ARTN), astrogliosis, c-Jun N-terminal kinase (JNK), extracellular signal-regulated kinase (ERK), hypoxic- ischemia injury, immature retina, neuroinflammation

Posted Date: December 1st, 2020

DOI: https://doi.org/10.21203/rs.3.rs-115996/v1

License: (9) This work is licensed under a Creative Commons Attribution 4.0 International License.

Read Full License 


\section{Abstract}

Background: Hypoxic-ischemia $(\mathrm{HI})$ is a major cause of acquired visual impairment in children of developed countries. Previous studies have shown that systemic administration of 7,8-dihydroxyflavone (DHF), a selective tropomyosin receptor kinase B (TrkB) agonist, provided long-term neuroprotection against $\mathrm{HI}$ injury in immature retina. However, the target genes and the mechanisms of the neuroprotective effects of TrkB signaling are not known.

Methods: $\mathrm{HI}$ retinal injury was induced by unilateral common carotid artery ligation followed by $8 \%$ oxygen for $2 \mathrm{hrs}$ at P7 rat pups. DHF was administered intraperitoneally at $2 \mathrm{hrs}$ before and $18 \mathrm{hrs}$ after $\mathrm{HI}$ injury. Polymerase chain reaction (PCR) array was used to identify genes upregulated after DHF treatment, then confirmed with quantitative real-time reverse transcriptase PCR and Western blot. Effects of the downstream mediator of DHF were assessed by intravitreal injection of neutralizing antibody at 4 hrs after DHF administration (24 hrs after $\mathrm{HI}$ ). Meanwhile, the target protein was injected into the vitreous at $24 \mathrm{hrs}$ after $\mathrm{HI}$ to validate its protective effect when exogenously supplemented. The outcomes were assessed by electroretinography and by histopathological sections of the rat retina.

Results: Systemic DHF treatment after HI significantly increased the expression of artemin (ARTN) gene and protein at P8 and P10, respectively. The neuroprotective effects of DHF were inhibited after the blockade of ARTN protein with an increase in neuroinflammation and astrogliosis. ARTN treatment showed long-term protection against $\mathrm{HI}$ injury at both the histopathological and functional levels. The neuroprotective effects of ARTN were related to a decrease in microglial activation at P17, and attenuation of astrogliosis at P29. ARTN enhances phosphorylation of RET, ERK, and JNK, but not AKT or p38 in the immature retina.

Conclusions: Neuroprotective effect of TrkB agonist is partially exerted through a mechanism that involves ARTN because the protective effect is ameliorated by ARTN sequestration. ARTN treatment after $\mathrm{HI}$ injury protects the immature retina by attenuating the late neuroinflammation and astrogliosis in the immature retina via ARTN/RET/JNK/ERK signaling pathway. ARTN can be a strategy to provide long-term protection in immature retina against $\mathrm{HI}$ injury.

\section{Introduction}

With the advances in perinatal care, the survival rates for infants with hypoxic-ischemic encephalopathy have increased [1]. Up to $60 \%$ of infants who survive have severe disabilities including mental retardation, epilepsy, and cerebral palsy [2]; however, hypoxic-ischemia $(\mathrm{HI})$ is also a major cause of acquired visual impairment in children of developed countries [3,4]. Although cortical visual dysfunction is an important cause of visual impairment, our previous studies have demonstrated that the immature retina was also susceptible to $\mathrm{HI}$ injury $[5,6]$. Compared with the adult rodents, $\mathrm{HI}$ caused more rapid and extensive damage of the retina at both the histopathological and functional levels in the rat pups, involving prominent neuroinflammation with astrogliosis, and caspase-dependent apoptotic neuronal deaths $[5,6]$. 
The neuroprotective role of brain-derived neurotrophic factor (BDNF) in the retina has extensively been tested over the past two decades [7]. Through the activation of tropomyosin receptor kinase B (TrkB), BDNF leads to increased neurogenesis, neuronal survival, and differentiation[8]. However, the use of BDNF as a treatment for retinal degenerations has not been successful due to the challenges in sustaining adequate therapeutic levels [9]. The use of BDNF mimetics has thus been investigated as an alternative treatment against neuronal injury[10]. In a previous study, we showed that in rat pups, the systemic administration of 7,8-dihydroxyflavone (DHF), a selective TrkB agonist, provided long-term protection against retinal $\mathrm{HI}$ injury at both the histological and functional levels, and was related to decreased astrogliosis and increased neurogenesis [11]. Interestingly, DHF treatment did not decrease the apoptosis, inflammation, blood-retina-barrier damage, and cell loss in the inner retina at the early stages of $\mathrm{HI}$ injury. Other than the activation of extracellular signal-regulated kinase (ERK), the initial target gene and the downstream mediators triggered by DHF leading to the late neurogenesis after $\mathrm{HI}$ are mostly unknown. In clinical practice, most treatments are intervened after the onset of disease, such as acute retinal ischemia [12], diabetic retinopathy [13], and glaucoma [14]. At that stage, the retinal neurons are undergoing inflammation and entering cell death. Therefore, it is important to find a strategy which triggers late neurogenesis to restore visual function at the stage after early inflammation and cell loss. In this study, we used a quantitative real-time polymerase chain reaction (qRT-PCR) array to elucidate the subsequent gene expression involved in the DHF-mediated neuroprotection and evaluated the protective effects and mechanism of the target protein in the immature retina after $\mathrm{HI}$ injury

\section{Materials And Methods}

\section{Animals}

This study was approved by the Animal Care Committee and the Ethics committee of Chang Gung Memorial Hospital in Kaohsiung. Ten to twelve Sprague-Dawley rat pups per dam were used and housed with a 12/12 hour (hr) light/dark schedule in a temperature- and humidity-controlled colony room. The pups were housed with their dams until weaning at postnatal $(P)$ day 21 and then housed in groups of 4 to 5 per cage.

\section{Hypoxic-ischemia eye injury}

At P7, the animals were anesthetized with $2.5 \%$ halothane (balance, room air), then the right common carotid artery was surgically exposed and permanently ligated. After surgery, the pups were returned to the dam for $1 \mathrm{hr}$, then placed in air-tight containers through which humidified $3 \mathrm{~L} / \mathrm{min} 8 \%$ oxygen (balance, nitrogen) was maintained for $2 \mathrm{hrs}$ [6]. The sham controls underwent anesthesia and surgical exposure but did not receive artery ligation and were not placed in a hypoxic chamber.

\section{Systemic DHF treatment}


Two hours before and $18 \mathrm{hrs}$ after the induction of $\mathrm{HI}$, the rat pups were injected intraperitoneally with either DHF (5 mg/kg; Tokyo Chemical Industry Co., Tokyo, Japan) or dimethyl sulfoxide (DMSO; 10\%; Sigma-Aldrich Corp., St. Louis, MO, USA).

\section{PCR array}

The Rat Neurogenesis RT ${ }^{2}$ Profiler ${ }^{\mathrm{TM}}$ PCR Array (Qiagen, Catalog \# PARN-404Z, Maryland, USA), which consisted of primers for 84 genes related to neurogenesis and neural stem cells was used for gene expression analysis. The rats treated with either DHF or DMSO were sacrificed, and total RNA was prepared from the retinas at $\mathrm{P} 10$ and immediately froze at $-70^{\circ} \mathrm{C}$. Aliquots of $1 \mu \mathrm{g}$ RNA per retina were reverse-transcribed using a $\mathrm{RT}^{2}$ First Strand Kit (Qiagen). The complementary DNA (cDNA) was mixed with SYBR Green (Qiagen) into the array plates, and cycling was performed according to the manufacturer's protocol. The data obtained from the array were normalized using multiple housekeeping genes and analyzed by comparing $2^{-\triangle \mathrm{Ct}}$ of the normalized data. Fold changes were calculated relative to retinal extracts from $\mathrm{HI}$ animals treated with DHF and DMSO. The results were confirmed by quantitative Real-time reverse transcriptase polymerase chain reaction (qRT-PCR) analysis on the individual samples for genes that showed the strongest upregulation and downregulation.

\section{Quantitative real-time reverse transcriptase polymerase chain reaction}

Retinas were dissected and grounded with a mortar and pestle in liquid nitrogen under RNase-free conditions. Total RNA was extracted using TRIzol reagent (Invitrogen, Carlsbad, CA, USA). Aliquots of $5 \mu \mathrm{g}$ total RNA were reverse-transcribed to cDNA using SuperScript III Reverse Transcriptase (Invitrogen). The cDNA was amplified by PCR using the following gene-specific primers: ARTN, 5'-

CAGAGCCTGGAAAGATGACC-3' (forward) and 5'-AGAGCTGGGATCCATGAACA-3' (reverse); and glyceraldehyde 3-phos-phate dehydrogenase (GAPDH), 5'-TCTTGTGCAGTGCCAGCCTC-3' (forward) and 5'-GTCACAAGAGAAGGCAGCCCTGG-3' (reverse). Template was amplified at $95^{\circ} \mathrm{C}$ for 5 minutes, followed by 45 cycles of PCR at $95^{\circ} \mathrm{C}$ for 10 seconds, $60{ }^{\circ} \mathrm{C}$ for 20 seconds, and at $72{ }^{\circ} \mathrm{C}$ for 20 seconds using LightCycler ${ }^{\circledR} 480$ SYBR Green I Master (Roche, Indianapolis, IN, USA) and LightCycler ${ }^{\circledR} 480$ instrument (Roche) for analyzing ARTN and GAPDH. The $\mathrm{C}_{t}$ assigned as the beginning of logarithmic amplification was computed by the software program of the equipment (Roche). The relative expression level was defined as $2^{-\Delta C t}$, where $\Delta C_{t}=C_{t}$ target gene $-C_{t \beta \text {-actin }}$. The fold changes in mRNA expression is defined as $2^{-\triangle \Delta C t}$, where $\triangle \Delta C t=\Delta C_{t \text { treatment }}-\Delta C_{\text {t vehicle. }}$

\section{Investigation of the effects of artemin}


For evaluating whether the protective effect of DHF come from upregulating endogenous ARTN, intravitreal injection of either ARTN-neutralizing antibody (ARTN Ab, 1 mg; R\&D systems, Minneapolis, MN, USA) [15] or phosphate buffered saline (PBS) was performed at post-HI 24 hrs, which was 6 hrs after DHF treatment. For assessing the effect of exogenous ARTN in HI injured retinas, either ARTN (1 mg, Peprotech, Rocky Hill, NJ, USA) [16] or $\mathrm{H}_{2} \mathrm{O}$ was administered by intravitreal injection at post-HI $24 \mathrm{hrs}$. Animals received intraperitoneal injection of 5-bromo-2'-deoxyuridine (BrdU; 100 mg/kg; Sigma-Aldrich Corp.) for consecutive 3 days from P8 to P10 for identifying cell proliferation.

\section{Functional evaluation of the retina by electroretinography}

At P22 and P29, full-field scotopic flash electroretinograms (ERGs) (RETIport ERG; Roland Consult, Brandenburg, Germany), were recorded from both eyes of the rat pups as previously described [6]. Briefly, the pupils were topically dilated by $1 \%$ Tropicamide (Mydriacyl, Alcon, Puurs, Belgium) and 1\% cyclopentolate (Cyclogyl, Alcon, Puurs, Belgium), then the eyes were dark-adapted for $1 \mathrm{hr}$ before performing ERG. The animals were sedated by intramuscular injections of a mixture of Rompun (10 $\mathrm{mg} / \mathrm{kg}$; Bayer Korea, Seoul, Korea) and Zoletil-50 (25 mg/kg; Virbac, Carros, France), then a standard white flash on a dark background scotopic 0-dB ERG was recorded. The stimulus luminance was 3 $\mathrm{cds} / \mathrm{m}^{2}$ with a duration of $10 \mathrm{~ms}$. Responses from 20 identical flashes applied at 10-second intervals were averaged [5].

\section{Histological assessment of retinal injury}

Paraffin sections of the retina were dewaxed, hydrated through graded concentrations of alcohol, and placed in phosphate-buffered saline. Cryosections were prepared after fixation in $4 \%$ paraformaldehyde and dehydration in a sucrose gradient. Two sections per retina were randomly selected for hematoxylin and eosin staining. Images were acquired using a light microscope (Nikon, Tokyo, Japan). Retinal damage was quantified by assigning different grades: grade 0 , preserved retinal ganglion cell (RGC) and all retinal layers comparable to sham control; grade 1, moderate decrease in RGC counts and thickness of the inner plexiform layer (IPL); grade 2, complete loss of RGCs and IPL (Additional file 1).

\section{Immunohistochemical staining}

After antigen unmasking and blocking of nonspecific sites, the sections were incubated overnight at $4{ }^{\circ} \mathrm{C}$ with primary antibodies against ARTN (1:10; R\&D systems), phosphorylated (p)RET (1:10; Abcam, Cambridge, UK), ED1 (1:100; Biosource, Camarillo, CA, USA), antiglial fibrillary acidic protein (GFAP; 1:200; Millipore, Temecula, CA, USA), and BrdU (1:100; Novocastra, Newcastle upon Tyne, UK), then subsequently incubated with secondary antibodies for 60 minutes at room temperature. The immunoreactivity of ARTN was evaluated at a 200囚 magnification by calculating the integrated optical density (IOD) with the ImagePro Plus 6.0 software [11]. The number of ED1 ${ }^{+}$and $\mathrm{Brdu}^{+}$cells were counted 
in an area of $400 \times 100 \mu \mathrm{m}$ at $200 \mathrm{X}$ magnification. The ameboid ED $1^{+}$cells were defined as reactive microglial cells. GFAP immunoreactivity was quantified by assigning different grades: grade 1 , immunoreactivity in the nerve fiber layer (NFL) and around vessels; grade 2, immunoreactivity in the NFL in an outward tentacle-like pattern, extending toward the inner nuclear layer (INL); grade 3, showing occasional and grade 4, showing extensive GFAP immunoreactivity extending from the NFL to the outer nuclear layer (ONL) [11].

\section{Western blot Analysis}

Retinas were homogenized and $40 \mu \mathrm{g}$ samples were resolved by $10 \%$ sodium dodecyl sulfate polyacrylamide gel electrophoresis, then blotted to nitrocellulose membranes. Membranes were blocked with $5 \%$ nonfat dry milk, incubated with primary antibodies and horseradish-conjugated secondary antibodies, and the signal was visualized with enhanced chemiluminescence. The following primary antibodies were used: anti-ERK (1:10000; Cell Signaling Technology, Danvers, MA,USA), anti-pERK (1:2000; Millipore), anti- c-Jun N-terminal kinase (JNK, 1:2000; Cell Signaling Technology), anti-pJNK (1:1000; Cell Signaling Technology), anti-p38 (1:5000; Abcam), anti-pp38 (1:10000; Abcam), anti-Akt (1:10000; Cell Signaling Technology), and anti-pAkt (1:1000; Cell Signaling Technology). After densitometric analysis, data were normalized against GAPDH (Millipore) and the ratio of protein expression in the treated eyes to the sham controls was calculated.

\section{Statistics}

Statistical analyses were performed by 1-way ANOVA or Kruskal-Wallis test using GraphPad Prism 4 software (GraphPad, San Diego, CA, USA). Data were presented as mean \pm standard error. P values of $<0.05$ were considered statistically significant.

\section{Results}

\section{Systemic DHF treatment increases the expression of ARTN after $\mathrm{HI}$ in immature retinas}

Previously we showed that systemic DHF treatment was able to protect the immature retina against $\mathrm{HI}$ injury [11]. To investigate which are the mediators for the HI protective effects of DHF treatment in the immature retina, PCR array was performed at P10 and found that the expression of ARTN gene in the DHF-treated $\mathrm{HI}$ group was 2 times higher than that in the DMSO-treated HI group. In contrast, other neurotrophic factors including glial cell line-derived neurotrophic factor (GDNF) and BDNF were not significantly elevated in the DHF-treated HI group (Additional file 2). Using RT-PCR, we confirmed that ARTN mRNA levels in the DHF-treated HI group were significantly higher than the DMSO-treated HI group and the sham controls at $24 \mathrm{hrs}$ after HI (Fig. 1a). The immunohistochemical stain showed that ARTN 
protein was prominently expressed in the RGC, IPL, and INL of sham controls and the DHF-treated HI group at P8 but was markedly decreased in the DMSO-treated HI group (Fig. 1b). The ARTN immunoreactivity was also significantly lower in the DMSO-treated HI group compared to the DHF-treated $\mathrm{HI}$ group and the sham group at P10 (Fig. 1c; $P<0.05$ ).

\section{Intravitreal injection of ARTN-neutralizing antibody blocks the long-term neuroprotection of DHF against $\mathrm{HI}$ at both the functional and histopathological levels}

After $\mathrm{HI}$ injury, endogenous artemin was sequestered by intravitreal injections of artemin-neutralizing antibody (ARTN $A b$ ) to determine whether the neuroprotective effects of DHF in the immature retina was mediated by artemin (Fig. 2a). ERG performed at P22 and P29 showed that the a-wave (associated with rod photoreceptor activity) and b-wave (Müller glial and bipolar cells activity) amplitudes in the DHF-PBS group were relatively preserved, while in the DMSO-treated group and the DHF-ARTN Ab group, the amplitudes of the a-wave and b-wave were markedly decreased (Fig. 2b). Group data showed that the bwave amplitude in the DHF-ARTN Ab group was significantly lower $(P<0.05)$ than the DHF-PBS group but was similar to the DMSO-treated group (Fig. $2 b$ ). The a-waves amplitudes were not significantly different between the DHF-PBS, DHF-ARTN Ab, and DMSO-treated HI groups (data not shown).

Compatible with the functional alterations, there was almost a complete loss of the inner retinal layers, including the RGC, IPL, and INL, in addition to a partial loss of the outer retina layers, including the outer plexiform layer (OPL) and the ONL in the DMSO-treated HI group at P16 and P29 (Fig. 2C). Although in general thinner than the controls, the retinal layers in the DHF-PBS group were relatively preserved after HI. In contrast, the DHF-ARTN Ab group showed severe inner retinal damages after HI, especially at P29. Semiquantitative data showed that there were no significant differences in the severity of retinal damages between the DMSO-, DHF-PBS- and DHF-ARTN Ab-treated HI group at P16. However, at P29, the DMSO- and the DHF-ARTN Ab-treated groups showed significantly more severe retinal damages than the DHF-PBS group (Fig. 2c, P< 0.05). There were no significant differences between the DMSO- and DHFARTN Ab-treated HI groups. These data suggest that the neuroprotective effects of DHF are largely mediated by ARTN and that ARTN was involved in the long-term neuroprotective effects of DHF against $\mathrm{HI}$ retinal injury.

\section{Blockade of ARTN abolishes the neuroprotection of DHF through an increase in neuroinflammation and astrogliosis}

In our previous study, we also showed that the long-term neuroprotective effects of DHF were related to increased neurogenesis and decreased astrogliosis [11]. Immunohistochemical staining showed that at P17, both the DHF-PBS and DHF-ARTN Ab groups had significantly increased Brdu ${ }^{+}$cells in the inner 
retina than the DMSO-treated $\mathrm{HI}$ groups (Fig. 3a, $\mathrm{P}<0.05$ ), suggesting that the blockade of ARTN did not decrease the neuronal proliferative effects of DHF treatment.

The GFAP immunoreactivity, indicating the presence of astrogliosis, in the DHF-PBS group was significantly lower than the DMSO-treated HI groups at P29. However, the DHF-ARTN Ab group had extensive GFAP immunostaining throughout all of the retinal layers, similar to the DMSO-treated HI group, and was higher than the DHF-PBS group (Fig. 3b, $\mathrm{P}<0.05$ ).

At $\mathrm{P} 17$, reactive microglial cells, or active $\mathrm{ED} 1^{+}$cells, were present in the inner retina of all of the groups other than the control. However, the number of $\mathrm{ED} 1^{+}$cells were similar between the DMSO-treated $\mathrm{HI}$ group and the DHF-ARTN Ab group and were both significantly higher than the DHF-PBS group (Figs. 3c, $P<0.05)$. These data suggest that the neuroprotective mechanisms of DHF mediated by ARTN involves modulation of astrogliosis and neuroinflammation, but not neurogenesis.

\section{Intravitreal injection of ARTN provides long-term protection against $\mathrm{HI}$ injury at the histopathological and functional levels in immature retinas}

To verify whether ARTN does provide protection against $\mathrm{HI}$ injury or not, the rat pups received intravitreal injection of either ARTN or $\mathrm{H}_{2} \mathrm{O} 24 \mathrm{hrs}$ after $\mathrm{HI}$ injury. The ERG at P22 and P29 showed that the a-wave and b-wave in the ARTN-treated $\mathrm{HI}$ group were relatively preserved, with $\mathrm{b}$-wave amplitudes that were significantly higher than the $\mathrm{H}_{2} \mathrm{O}$-treated $\mathrm{HI}$ group (Fig. 4a, both $P<0.05$ ). In line with the functional alterations, despite the presence of damaged inner retinal layers in the ARTN-treated $\mathrm{HI}$ group, it was to a lesser degree than the $\mathrm{H}_{2} \mathrm{O}$-treated $\mathrm{HI}$ group and was statistically significant at both P16 and P29 (Fig. 4b, both $P<0.05)$.

\section{ARTN treatment protects the immature retina against HI injury by inhibiting late neuroinflammation and astrogliosis}

Immunohistochemical staining showed that at P17, the number of proliferating or Brdu ${ }^{+}$cells were not significantly different between the ARTN- and $\mathrm{H}_{2} \mathrm{O}$-treated $\mathrm{HI}$ groups (Fig. 5a). However, GFAP immunostaining at P29 demonstrated that ARTN treatment significantly decreased astrogliosis after $\mathrm{HI}$ (Fig. 5b; $P<0.05$ ). Both ARTN- and $\mathrm{H}_{2} \mathrm{O}$-treated $\mathrm{HI}$ groups showed significantly greater neuroinflammation, with increased active ED $1^{+}$cells, compared to the sham controls at P10 (Fig. 5 c; $P<$ 0.001). However, at P17, the number of active ED $1^{+}$cells in the ARTN-treated HI group was significantly decreased compared to the $\mathrm{H}_{2} \mathrm{O}$-treated group (Fig. $5 \mathrm{c} ; P<0.05$ ) and was similar to sham controls. These data confirm the prior results of ARTN blockade and suggest that ARTN protects the immature retina by decreasing astrogliosis and late neuroinflammation after $\mathrm{HI}$. 


\section{ARTN treatment after $\mathrm{HI}$ injury enhances RET, ERK, and JNK phosphorylation in immature retinas}

The ARTN-treated HI group had prominent ARTN immunostaining in the RGC and INL at P10, which indicates that ARTN continues to be expressed in the inner retina 2 days after the treatment. There were significant differences between the ARTN-treated $\mathrm{HI}$ group and the $\mathrm{H}_{2} \mathrm{O}$-treated $\mathrm{HI}$ group and sham controls (Fig. 6a, P < 0.01). Receptor tyrosine kinase RET is the main effecter of ARTN. RET phosphorylation (pRET) results in the activation of multiple downstream signaling. Immunostaining also showed that ARTN treatment after $\mathrm{HI}$ induces significantly increased RET phosphorylation (pRET) immunoreactivity in the RGC, IPL, and OPL (Fig. 6b, P<0.05). This suggests that ARTN exerts its neuroprotective effects through the activation of receptor tyrosine kinase RET.

To investigate the downstream signaling pathways of ARTN/RET, western blots were performed at P10. There were no significant differences in the total and phosphorylated p38 or AKT between the ARTN-and $\mathrm{H}_{2} \mathrm{O}$-treated $\mathrm{HI}$ groups at P10 (Figs. 6c-d). However, the ARTN-treated group had significantly increased phosphorylation of ERK and JNK than the $\mathrm{H}_{2} \mathrm{O}$-treated $\mathrm{HI}$ group (Figs. $6 \mathrm{e}-\mathrm{f}$, both $P<0.05$ ), which showed that the neuroprotective effects of ARTN are mediated through the ARTN/RET/JNK/ERK signaling pathway.

\section{Discussion}

DHF, a TrkB agonist, was previously shown to protect the immature retina against $\mathrm{HI}$ injury through promoting neurogenesis [11]. In this study, we aimed to investigate what is the target gene involved in the neuroprotective effects of DHF and were the first to demonstrate that after HI injury, ARTN expression in the immature retina is upregulated by systemic DHF treatment. We also demonstrated that ARTN treatment after $\mathrm{HI}$ injury protects the immature retina by attenuating the late neuroinflammation and astrogliosis in the immature retina. The ARTN/RET/JNK/ERK signaling pathway seems to be critically involved in the ARTN-mediated neuroprotection (Fig. 7). We therefore suggest that ARTN may be an alternative treatment for restoring retinal function after $\mathrm{HI}$ injury in neonates.

The BDNF/TrkB signaling pathways and their requirement in neuroprotection and neuron development are well-established, but how ARTN induce retinal protection is poorly understood. ARTN is a member of the glial cell line-derived neurotrophic factor (GDNF) family of ligands (GFLs, including GDNF, neurturin, artemin, and persephin), which form ternary complexes with the GDNF family receptor (GFRa). Assembling of the GFL-GFRa-RET(tyrosine kinase receptor) complex triggers the dimerization of RET, leading to autophosphorylation of specific tyrosine residues in its intracellular domain and subsequent activation of different intracellular signal cascades. These include AKT, ERK, JNK, P38, and Src, which are involved in the regulation of cell survival, differentiation, proliferation, migration, chemotaxis, morphogenesis, neurite outgrowth, and synaptic plasticity $[17,18]$. BDNF protect injured RGCs in vitro and in vivo, acting directly on RGCs that express TrkB [7]. Conversely, it has been demonstrated that GFLs 
does not enhance the survival of RGCs in vitro, despite that GFLs enhances RGC survival in vivo [19]. These results suggest that the GFLs does not act directly on RGCs to increase cell survival in vivo. However, the GFLs reduces glutamate-mediated excitotoxicity in axotomized RGCs related to increasing the expression of the glutamate/aspartate transporter-1 (GLAST-1) in retinal Müller glial cells (RMG) and astrocytes [8]. Previous findings are in general consistent with the findings in the present study, in which we found that increased ARTN levels, either induced by DHF treatment or exogenously supplemented by intravitreal administration, protects the immature retina against $\mathrm{HI}$ injury by ameliorating the activation of microglia and astrocytes, but does not promote neuronal regeneration.

In a way, it is surprising that ARTN, despite showing neuroprotective effects in the immature retina, does not promote neurogenesis as previously demonstrated by DHF treatment [11]. This suggests that DHF may trigger more than one neuroprotective pathway or target gene; one may involve upregulation of ARTN to decrease inflammation and astrogliosis as demonstrated in this study, while others may be involved in neurogenesis, but remains elusive and needs to be further investigated in future studies.

Recent evidence has proposed that neurotrophic rescue of retinal neurons can be indirect, mediated by the interaction of other neurotrophic factors with glial cells, which in turn release secondary factors acting directly on neurons [20]. BDNF has no direct effect on isolated photoreceptor cells. Thus, it may protect photoreceptors, at least partly, through RMG. BDNF-treated cultured RMG expressed BDNF, GDNF, and basic fibroblast growth factor (bFGF) to protect the RGC against glutamate toxicity [8]. RMG responded to Neurotrophin-3 or nerve growth factor (NGF), respectively, by increasing or decreasing their production of bFGF, which in turn results in either the protection or increased apoptosis of photoreceptor cells [21]. We previously demonstrated that systemic DHF treatment did not prevent early neuronal apoptosis, but enhanced the proliferation of RMG and bipolar cells and thereby restored the retinal function after $\mathrm{HI}$ injury in rat pups [11]. The present study further shows that the blockade of endogenous ARTN abolishes the long-term neuroprotective effects of DHF through increasing late neuroinflammation and astrogliosis. These data suggested that TrkB signaling involves not only RGC but also RMG, astrocytes, and microglia for neuroprotection. Therefore, a promising neuroprotective strategy may involve not only promoting neuronal survival but also involve the cross-talks of neurotrophic factors with which the microglia, RMG, and inner retinal neurons communicate during $\mathrm{HI}$ retinal injury.

ARTN is an important mediator of diverse physiological and pathophysiological functions, including the development and maintenance of diverse neuronal populations, neurite outgrowth, and nerve regeneration [18]. In the eye, ARTN is primarily expressed in the retina, and provides neuroprotection in the context of retinal degeneration $[16,20]$. ARTN has been used as a treatment of depression based on its effects on neuroplasticity [22]. ARTN has also been shown to trigger oncogenicity and metastasis by the activation of the AKT signaling pathway [23]. Recent evidence suggests that it may play a bi-directional role in the modulation of neuropathic pain and inflammation [24]. ARTN is increased by inflammation and mediates the nociceptive signaling in both humans and animals [17, 24, 25]. Anti-ARTN Ab treatment in a human neuroblastoma cell line completely inhibited ARTN/RET/ERK activation and block capsaicininduced calcitonin gene-related peptide secretion from primary cultures of rat dorsal root ganglia neurons 
[26]. Conversely, a phase 2 clinical trial showed evidence of pain relief by ARTN in patients with lumbosacral radiculopathy [27]. Although the exact mechanisms underlying the inflammation regulation by ARTN require further investigation, the findings of this study provide novel evidence that ARTN may exert neuroprotection via anti-inflammation action through the RET/JNK/ERK signaling pathway in the immature retina.

We found that ARTN post-treatment activates both ERK and JNK signaling pathways in the immature retina during $\mathrm{HI}$ injury. The GFLs and GFR communications in the retina activate distinct signaling cascades, including ERK, JNK, and AKT, but with different time courses and are often involved in complex cross-talk on multiple levels [20]. These signaling pathways involved in various cellular processes, including cell proliferation, differentiation, senescence, and apoptosis. The coordination between these pathways determines the cell's fate. Our previous data have shown that ERK activation is pivotal for the long-term neuroprotective effects of DHF treatment. The ERK signaling mediates the effects of TrkB on RMG proliferation and transdifferentiation [11]. Conversely, JNK contributed to the apoptotic response of neuronal cells, and the JNK-dependent apoptosis could be suppressed by the activation of ERK [28]. However, recent studies indicated that periostin, an adhesion molecule, enhances the migration and differentiation of mesenchymal stem cells via the JNK signaling pathway under inflammatory conditions [29]. Although the exact mechanisms underlying the neuroprotection of ARTN require further elucidation, the findings of this study indicate that intracellular signaling dynamics between ERK and JNK play a significant role during inflammation in $\mathrm{HI}$ retinal injury.

Post-ischemic neuroinflammation in the immature central nervous system is a key pathophysiological factor in the development of HI-related injury [30]. It is highly likely that this secondary inflammation augments damage in the early phase of evolving cell death [31]. The downstream mediators of inflammation-induced injury include induction of immune mediators, reactive oxygen and nitrogen species, excitotoxicity, mitochondrial impairment, and reduced vascular integrity [31]. Microglial infiltration and astrogliosis may persist up to 21 days and 2 months after $\mathrm{HI}$ insult in animal and human studies, respectively $[32,33]$, while secreting proinflammatory cytokines during evolving neural injury, which also have a key role in the chronic or secondary inflammation of $\mathrm{HI}$ injury [31]. Our results showed that ARTN played an important role in modulating the late inflammation during $\mathrm{HI}$ injury since ARTN posttreatment significantly attenuated microglial activation at P17 but not P10. ARTN was also shown to significantly decrease the late astrogliosis at P29. While the mechanisms need to be further investigated, these data imply that supplementing or enhancing levels of ARTN was able to block the secondary or chronic inflammation caused by microglia and astrocytes and may be a viable therapeutic target to improve neurodevelopmental outcomes after HI injury.

The advent of therapeutic hypothermia for neonatal $\mathrm{HI}$ injury allowed us to intervene and alter the course of this disease with improved rates of infant survival [34]. However, $40 \%$ of infants with severe $\mathrm{HI}$ injury still suffer from significant neurologic disability [35]. The combination of perinatal infection and $\mathrm{HI}$ insult causes greater brain injury and poorer response to therapeutic hypothermia [36]. These concerns highlight the need for adding alternative therapeutic strategies, especially targeting neuroinflammation, 
beyond hypothermia therapy for $\mathrm{HI}$ injury. Our data suggest that ARTN can be a promising candidate since it provides long-term protection in immature retina against $\mathrm{HI}$ injury by ameliorating late neuroinflammation.

\section{Conclusions}

The present study demonstrates that the upregulation of ARTN, either triggered by TrkB agonist or supplied by exogenous administration, could activate RTN/RET/JNK/ERK signaling pathway and provide neuroprotection against $\mathrm{HI}$ injury by attenuating the late neuroinflammation and astrogliosis in the immature retinas. Therefore, ARTN might represent a valuable target for developing strategies to improve retinal and neurodevelopmental outcomes after $\mathrm{HI}$ injury in neonates.

\section{Abbreviations}

HI: Hypoxic-ischemia; BDNF: brain-derived neurotrophic factor; DHF:7,8-dihydroxyflavone; TrkB: tropomyosin receptor kinase B; ARTN: artemin; GDNF: glial cell line-derived neurotrophic factor; GFLs: GDNF family of ligands; GFRa: GDNF family receptor; P: postnatal; hr: hour; i.p: intraperitoneally; i.v.i: intravitreal injection; ARTN Ab: ARTN-neutralizing antibody; ERG: electroretinography; ERK: extracellular signal-regulated kinase; JNK: c-Jun N-terminal kinase; RGC: retinal ganglion cell; IPL: inner plexiform layer; NFL: nerve fiber layer; INL: inner nuclear layer; ONL: outer nuclear layer; GFAP: anti-glial fibrillary acidic protein; IOD: integrated optical density; RMG: retinal Müller glial cell.

\section{Declarations}

\section{Ethics approval and consent to participate}

We declare that we obtained the ethics approval by the Animal Care Committee at Chang Gung Memorial Hospital in Kaohsiung and by the Taiwan Ministry of Science and Technology (project nr, 106-2314-B182A-047).

\section{Consent for publication}

Not applicable

\section{Availability of data and materials}

The datasets used and/or analyzed during the current study are available from the corresponding author on reasonable request.

\section{Competing interests}


We declare that all co-authors have no competing interests for this study.

\section{Funding}

This work was supported by the grant from the Ministry of Science and Technology (Grant 106-2314-B182A-047; Taipei, Taiwan)

\section{Authors' contributions}

$\mathrm{HMH}$ designed the research, planned and performed the experiments, analyzed the data, and drafted the manuscript; $\mathrm{CCH}$ designed a part of the research, and collaborated in performing the experiments; LYCP analyzed the data and revised the manuscript; YCC designed the research project, analyzed the data, and revised the manuscript. The authors read and approved of the final manuscript.

\section{Acknowledgments}

None

\section{References}

1. Lee AC, Kozuki N, Blencowe H, Vos T, Bahalim A, Darmstadt GL, Niermeyer S, Ellis M, Robertson NJ, Cousens S, Lawn JE. Intrapartum-related neonatal encephalopathy incidence and impairment at regional and global levels for 2010 with trends from 1990. Pediatr Res. 2013;74 Suppl 1:50-72.

2. Pierrat V, Haouari N, Liska A, Thomas D, Subtil D, Truffert P, Groupe d'Etudes en Epidemiologie P. Prevalence, causes, and outcome at 2 years of age of newborn encephalopathy: population based study. Arch Dis Child Fetal Neonatal Ed. 2005;90:F257-261.

3. Cioni G, Fazzi B, Coluccini M, Bartalena L, Boldrini A, van Hof-van Duin J. Cerebral visual impairment in preterm infants with periventricular leukomalacia. Pediatric Neurology. 1997;17:331-338.

4. Lanzi G, Fazzi E, Uggetti C, Cavallini A, Danova S, Egitto MG, Ginevra F, Salati R, Bianchi PE. Cerebral Visual Impairment in Periventricular Leukomalacia. Neuropediatrics. 1998;29:145-150.

5. Huang HM, Huang CC, Wang FS, Hung PL, Chang YC. Activating the Wnt/ $\beta$-Catenin Pathway Did Not Protect Immature Retina from Hypoxic-Ischemic Injury. Invest Ophthalmol Vis Sci. 2015;56:4300.

6. Huang HM, Huang CC, Hung PL, Chang YC. Hypoxic-ischemic retinal injury in rat pups. Pediatr Res. 2012;72:224-231.

7. Afarid M, Torabi-Nami M, Zare B. Neuroprotective and restorative effects of the brain-derived neurotrophic factor in retinal diseases. J Neurol Sci. 2016;363:43-50.

8. Harada C, Guo X, Namekata K, Kimura A, Nakamura K, Tanaka K, Parada LF, Harada T. Glia- and neuron-specific functions of TrkB signalling during retinal degeneration and regeneration. Nat 
Commun. 2011;2:189.

9. Daly C, Ward R, Reynolds AL, Galvin O, Collery RF, Kennedy BN. Brain-Derived Neurotrophic Factor as a Treatment Option for Retinal Degeneration. Adv Exp Med Biol. 2018;1074:465-471.

10. Jang SW, Liu X, Yepes M, Shepherd KR, Miller GW, Liu Y, Wilson WD, Xiao G, Blanchi B, Sun YE, Ye K. A selective TrkB agonist with potent neurotrophic activities by 7,8-dihydroxyflavone. Proc Natl Acad Sci U S A. 2010;107:2687-2692.

11. Huang HM, Huang CC, Tsai MH, Poon YC, Chang YC. Systemic 7,8-Dihydroxyflavone Treatment Protects Immature Retinas Against Hypoxic-Ischemic Injury via Müller Glia Regeneration and MAPK/ERK Activation. Invest Ophthalmol Vis Sci. 2018;59:3124.

12. Biousse V, Nahab F, Newman NJ. Management of Acute Retinal Ischemia. Ophthalmology. 2018;125:1597-1607.

13. Wang W, Lo A. Diabetic Retinopathy: Pathophysiology and Treatments. Int J Mol Sci. 2018;19:1816.

14. Guymer C, Wood JPM, Chidlow G, Casson RJ. Neuroprotection in glaucoma: recent advances and clinical translation. Clin Experiment Ophthalmol. 2019;47:88-105.

15. DeBerry JJ, Saloman JL, Dragoo BK, Albers KM, Davis BM. Artemin Immunotherapy Is Effective in Preventing and Reversing Cystitis-Induced Bladder Hyperalgesia via TRPA1 Regulation. J Pain. 2015;16:628-636.

16. Omodaka K, Kurimoto T, Nakamura O, Sato K, Yasuda M, Tanaka Y, Himori N, Yokoyama Y, Nakazawa T. Artemin augments survival and axon regeneration in axotomized retinal ganglion cells. J Neurosci Res. 2014;92:1637-1646.

17. Nencini S, Ringuet M, Kim DH, Greenhill C, Ivanusic JJ. GDNF, Neurturin, and Artemin Activate and Sensitize Bone Afferent Neurons and Contribute to Inflammatory Bone Pain. J Neurosci. 2018;38:4899-4911.

18. Wong LE, Gibson ME, Arnold HM, Pepinsky B, Frank E. Artemin promotes functional long-distance axonal regeneration to the brainstem after dorsal root crush. Proc Natl Acad Sci U S A. 2015;112:6170-6175.

19. Koeberle PD, Ball AK. Neurturin enhances the survival of axotomized retinal ganglion cells in vivo: combined effects with glial cell line-derived neurotrophic factor and brain-derived neurotrophic factor. Neuroscience. 2002;110:555-567.

20. Hauck SM, Kinkl N, Deeg CA, Swiatek-de Lange M, Schoffmann S, Ueffing M. GDNF family ligands trigger indirect neuroprotective signaling in retinal glial cells. Mol Cell Biol. 2006;26:2746-2757.

21. Harada C, Azuchi Y, Noro T, Guo X, Kimura A, Namekata K, Harada T. TrkB Signaling in Retinal Glia Stimulates Neuroprotection after Optic Nerve Injury. Am J Pathol. 2015;185:3238-3247.

22. Di Cesare Mannelli L, Vivoli E, Salvicchi A, Schiavone N, Koverech A, Messano M, Nicolai R, Benatti P, Bartolini A, Ghelardini C. Antidepressant-like effect of artemin in mice: a mechanism for acetyl-Lcarnitine activity on depression. Psychopharmacology (Berl). 2011;218:347-356. 
23. Hezam K, Jiang J, Sun F, Zhang X, Zhang J. Artemin promotes oncogenicity, metastasis and drug resistance in cancer cells. Rev Neurosci. 2018;29:93-98.

24. Ikeda-Miyagawa Y, Kobayashi K, Yamanaka H, Okubo M, Wang S, Dai Y, Yagi H, Hirose M, Noguchi K. Peripherally increased artemin is a key regulator of TRPA1/V1 expression in primary afferent neurons. Mol Pain. 2015;11:8.

25. Thornton P, Hatcher JP, Robinson I, Sargent B, Franzen B, Martino G, Kitching L, Glover CP, Anderson $D$, Forsmo-Bruce $\mathrm{H}$, et al. Artemin-GFRalpha3 interactions partially contribute to acute inflammatory hypersensitivity. Neurosci Lett. 2013;545:23-28.

26. Jeong DG, Park WK, Park S. Artemin activates axonal growth via SFK and ERK-dependent signalling pathways in mature dorsal root ganglia neurons. Cell Biochem Funct. 2008;26:210-220.

27. Backonja M, Williams L, Miao X, Katz N, Chen C. Safety and efficacy of neublastin in painful lumbosacral radiculopathy: a randomized, double-blinded, placebo-controlled phase 2 trial using Bayesian adaptive design (the SPRINT trial). Pain. 2017;158:1802-1812.

28. Clark A. Psychiatric Diagnoses and Informed Consent. J Clin Ethics. 2018;29:93-99.

29. Tang Y, Liu L, Wang P, Chen D, Wu Z, Tang C. Periostin promotes migration and osteogenic differentiation of human periodontal ligament mesenchymal stem cells via the Jun amino-terminal kinases (JNK) pathway under inflammatory conditions. Cell Prolif. 2017;50.

30. Borjini N, Sivilia S, Giuliani A, Fernandez M, Giardino L, Facchinetti F, Calza L. Potential biomarkers for neuroinflammation and neurodegeneration at short and long term after neonatal hypoxicischemic insult in rat. J Neuroinflammation. 2019;16:194.

31. Hagberg H, Mallard C, Ferriero DM, Vannucci SJ, Levison SW, Vexler ZS, Gressens P. The role of inflammation in perinatal brain injury. Nat Rev Neurol. 2015;11:192-208.

32. Galinsky R, Lear CA, Dean JM, Wassink G, Dhillon SK, Fraser M, Davidson JO, Bennet L, Gunn AJ. Complex interactions between hypoxia-ischemia and inflammation in preterm brain injury. Dev Med Child Neurol. 2018;60:126-133.

33. Galinsky R, Draghi V, Wassink G, Davidson JO, Drury PP, Lear CA, Gunn AJ, Bennet L. Magnesium sulfate reduces EEG activity but is not neuroprotective after asphyxia in preterm fetal sheep. $J$ Cereb Blood Flow Metab. 2017;37:1362-1373.

34. Higgins RD, Raju T, Edwards AD, Azzopardi DV, Bose CL, Clark RH, Ferriero DM, Guillet R, Gunn AJ, Hagberg $\mathrm{H}$, et al. Hypothermia and other treatment options for neonatal encephalopathy: an executive summary of the Eunice Kennedy Shriver NICHD workshop. J Pediatr. 2011;159:851-858 e851.

35. Shankaran S. Therapeutic hypothermia for neonatal encephalopathy. Curr Opin Pediatr. 2015;27:152157.

36. Osredkar D, Thoresen M, Maes E, Flatebo T, Elstad M, Sabir H. Hypothermia is not neuroprotective after infection-sensitized neonatal hypoxic-ischemic brain injury. Resuscitation. 2014;85:567-572.

\section{Figures}




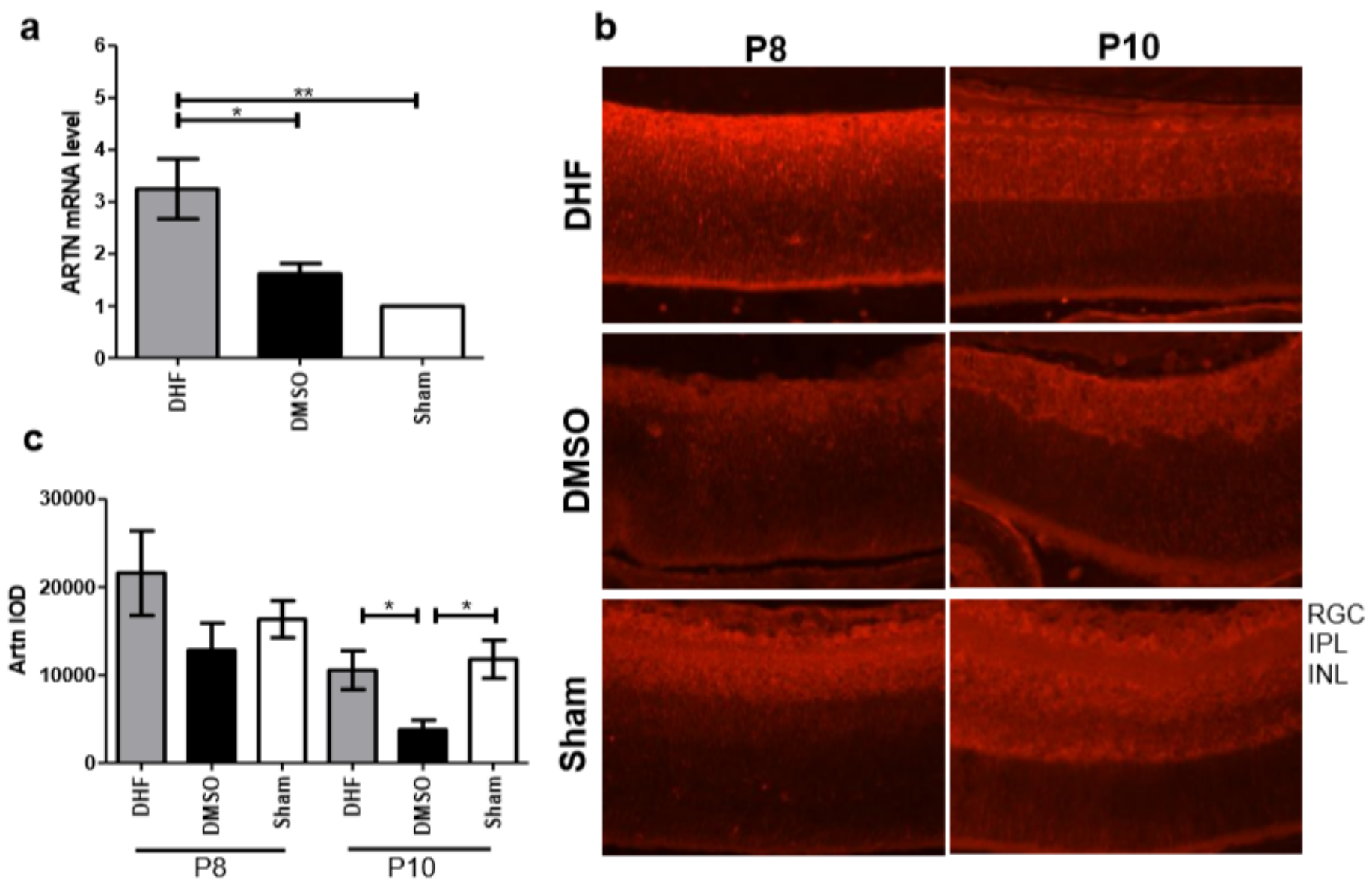

Figure 1

Systemic TrkB agonist- DHF increases the ARTN expression in HI Injured immature retina. (a) At P8, the ARTN mRNA level significantly increased in the DHF-treated HI group as compared with the DMSO-treated $\mathrm{HI}$ and sham control groups. (b) In the DHF-treated $\mathrm{HI}$ and sham control groups, the immunoreactivity of ARTN was prominent in the RGC, IPL, and INL at P8 and the expression decreased at P10. (c) The group data showed that the immunoreactivity of ARTN protein markedly increased in the DHF-treated HI group but decreased in the DMSO-treated HI group at $\mathrm{P} 10 * \mathrm{P}<0.05, * \star \mathrm{P}<0.01, \star \star \star P<0.001$. 


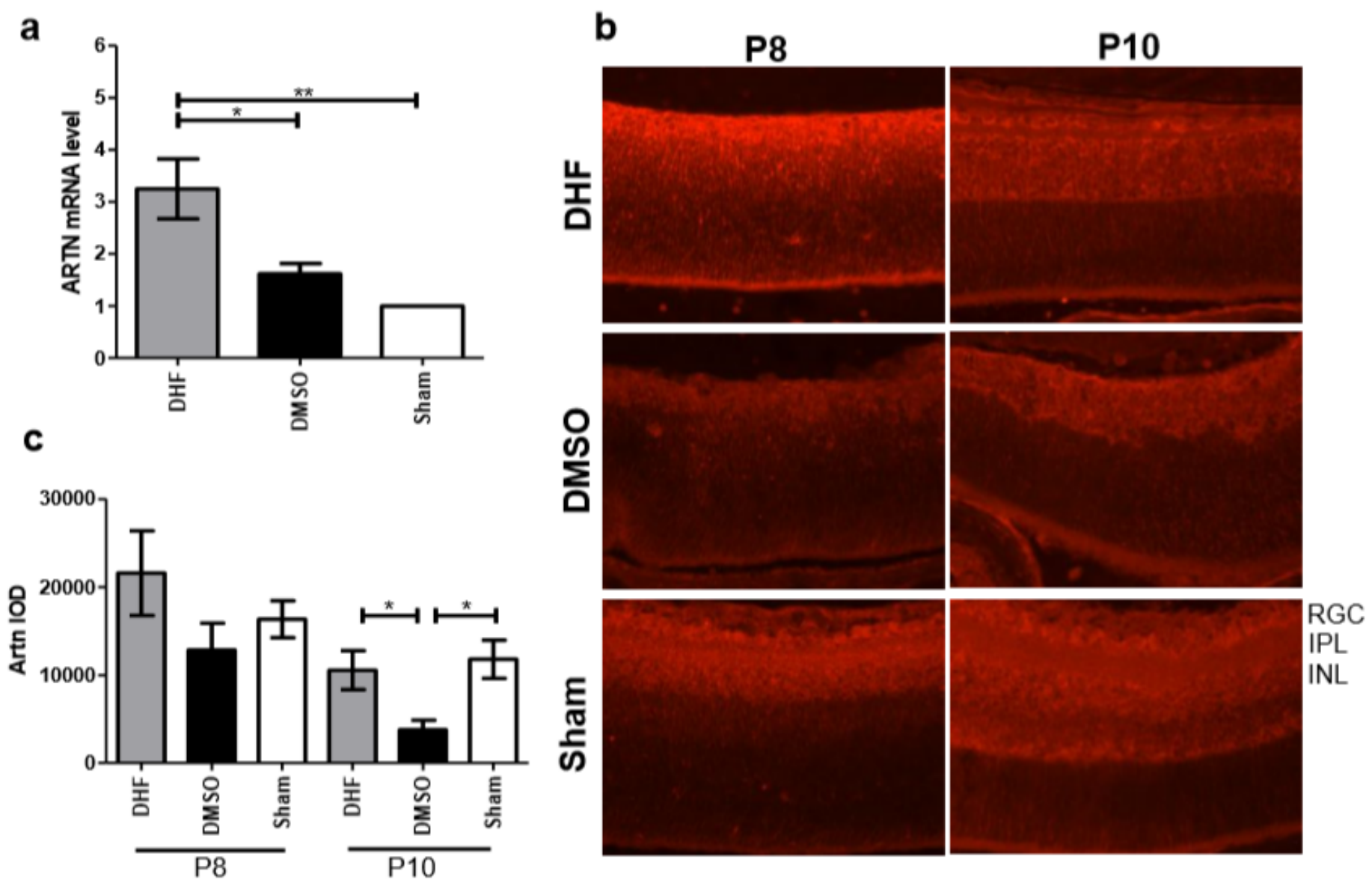

Figure 1

Systemic TrkB agonist- DHF increases the ARTN expression in HI Injured immature retina. (a) At P8, the ARTN mRNA level significantly increased in the DHF-treated HI group as compared with the DMSO-treated $\mathrm{HI}$ and sham control groups. (b) In the DHF-treated $\mathrm{HI}$ and sham control groups, the immunoreactivity of ARTN was prominent in the RGC, IPL, and INL at P8 and the expression decreased at P10. (c) The group data showed that the immunoreactivity of ARTN protein markedly increased in the DHF-treated HI group but decreased in the DMSO-treated HI group at $\mathrm{P} 10 * \mathrm{P}<0.05, * \star \mathrm{P}<0.01, \star \star \star P<0.001$. 


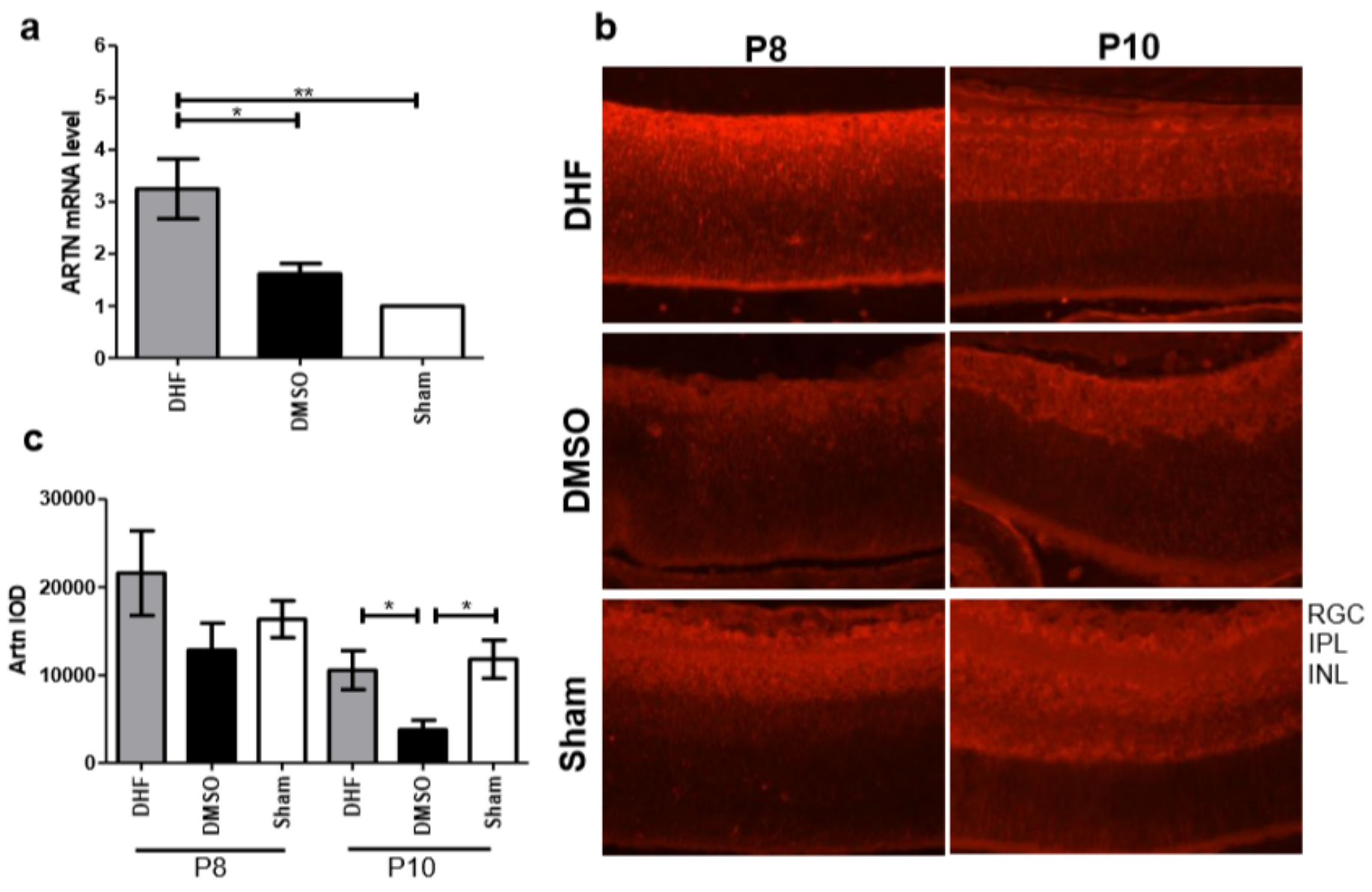

Figure 1

Systemic TrkB agonist- DHF increases the ARTN expression in HI Injured immature retina. (a) At P8, the ARTN mRNA level significantly increased in the DHF-treated HI group as compared with the DMSO-treated $\mathrm{HI}$ and sham control groups. (b) In the DHF-treated $\mathrm{HI}$ and sham control groups, the immunoreactivity of ARTN was prominent in the RGC, IPL, and INL at P8 and the expression decreased at P10. (c) The group data showed that the immunoreactivity of ARTN protein markedly increased in the DHF-treated HI group but decreased in the DMSO-treated HI group at $\mathrm{P} 10 * \mathrm{P}<0.05, * \star \mathrm{P}<0.01, \star \star \star P<0.001$. 
a
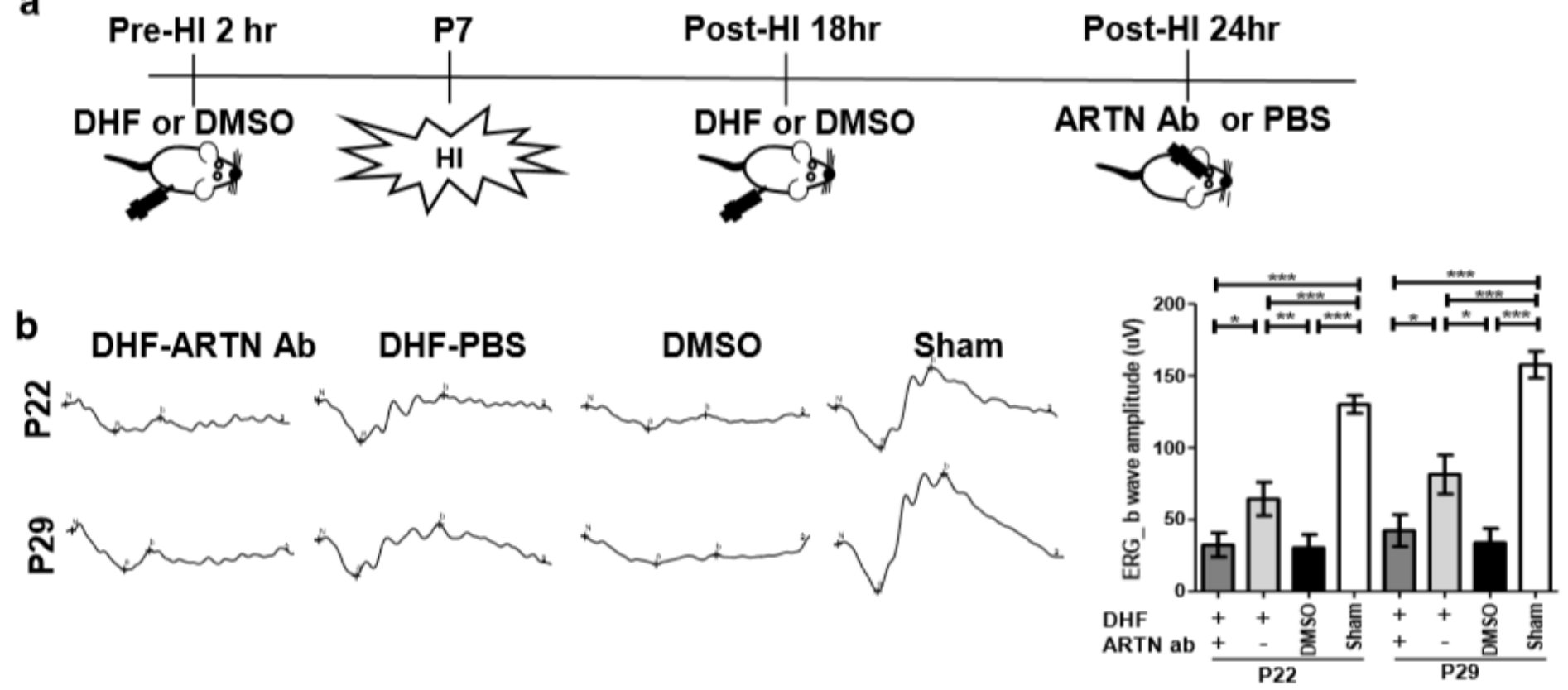

C
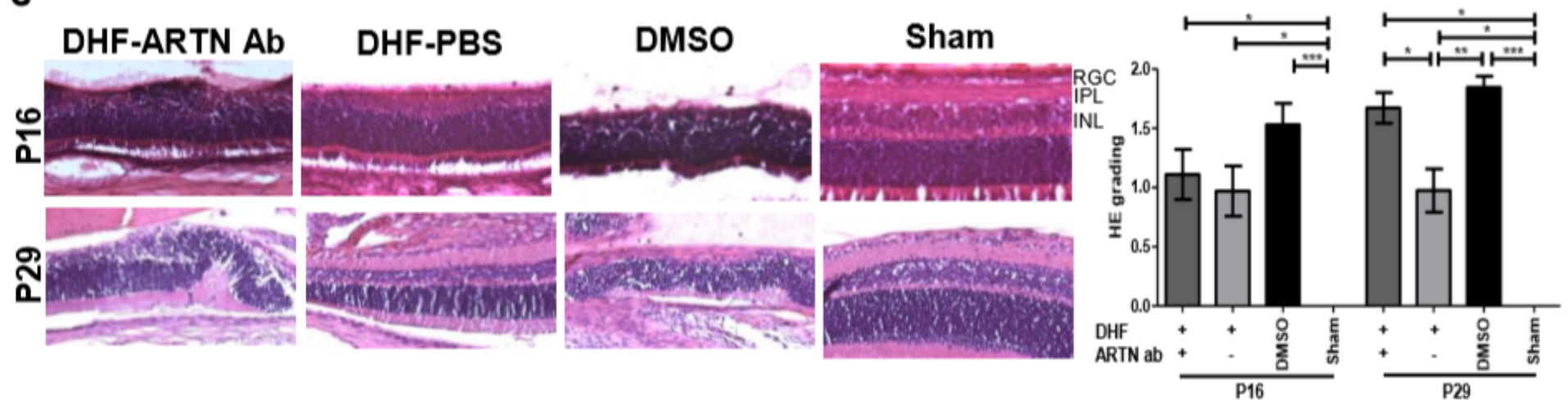

Figure 2

Intravitreal injection of ARTN Antibody inhibits the functional and histological protection of systemic DHF in the HI-injured retinas. (a) Two hours (hrs) before and $18 \mathrm{hrs}$ after the HI, either DHF or DMSO was injected peritoneally. ARTN- Antibody (Ab) or PBS was injected intravitreally at post-HI $24 \mathrm{hrs}$. (b) The retinal function evaluated by ERG demonstrated markedly depressed b-wave amplitudes in the $\mathrm{HI}$ injured groups at P22 and P29 as compared with sham controls. The group data showed the b-wave amplitude in the DHF-PBS group was significantly higher than the DHF-ARTN Ab and DMSO-treated groups after HI at P22 and P29. (c) Representative retinal histologic sections showed the number of RGCs and the thickness of IPL and INL decreased after HI at P16 and P29. The group data showed the grades of retinal damage were significantly higher in the HI groups than sham controls at P16 and P29. There were no significant differences between the DHF-PBS, DMSO, and DHF-ARTN Ab groups after HI injury at P16. As compared with the DHF-PBS group, the DMSO and DHF-ARTN Ab groups had increased retinal damage grades at $P 29 . * P<0.05, * \star P<0.01, * \star \star P<0.001$. 
a
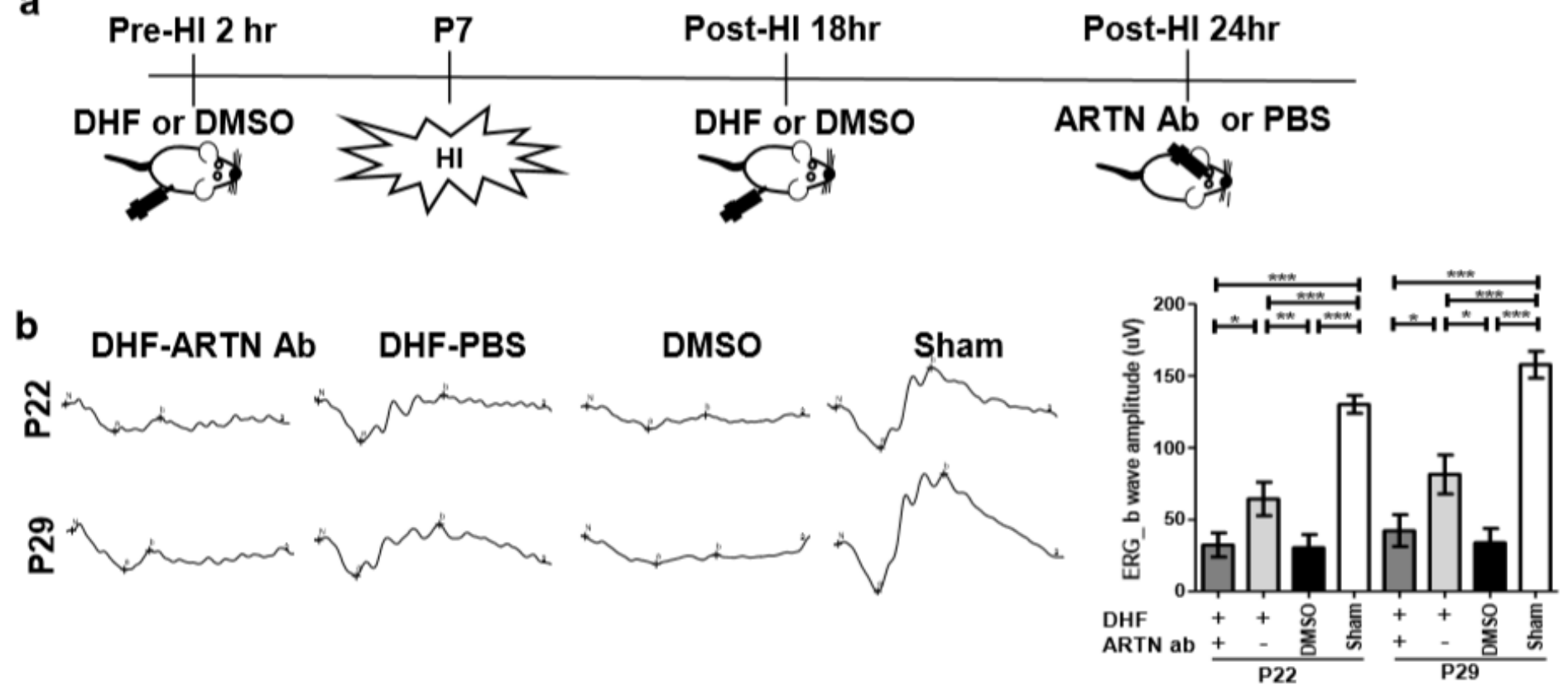

C
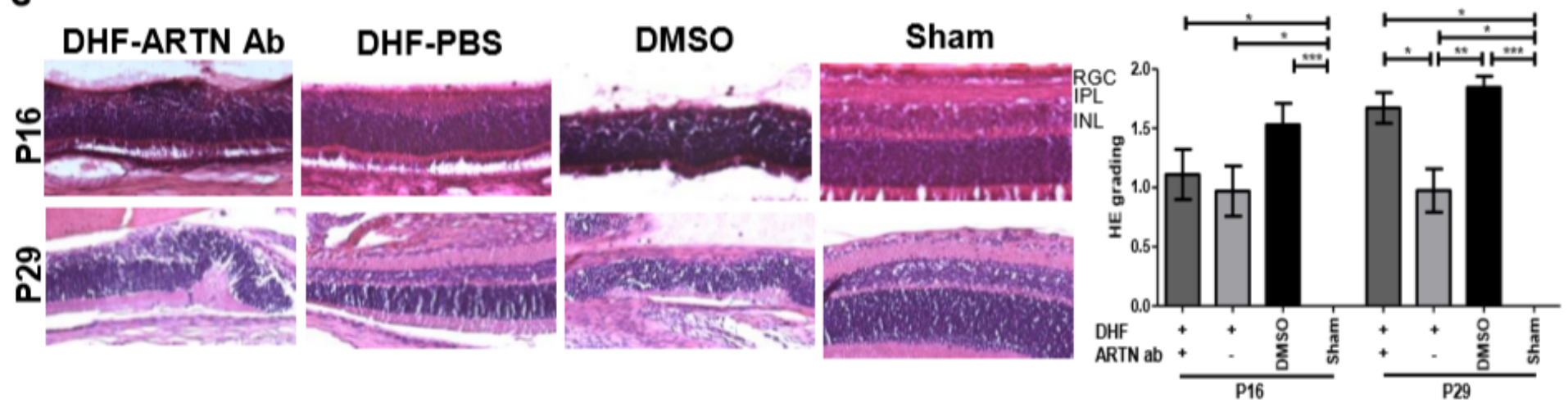

Figure 2

Intravitreal injection of ARTN Antibody inhibits the functional and histological protection of systemic DHF in the HI-injured retinas. (a) Two hours (hrs) before and $18 \mathrm{hrs}$ after the HI, either DHF or DMSO was injected peritoneally. ARTN- Antibody (Ab) or PBS was injected intravitreally at post-HI $24 \mathrm{hrs}$. (b) The retinal function evaluated by ERG demonstrated markedly depressed b-wave amplitudes in the $\mathrm{HI}$ injured groups at P22 and P29 as compared with sham controls. The group data showed the b-wave amplitude in the DHF-PBS group was significantly higher than the DHF-ARTN Ab and DMSO-treated groups after HI at P22 and P29. (c) Representative retinal histologic sections showed the number of RGCs and the thickness of IPL and INL decreased after HI at P16 and P29. The group data showed the grades of retinal damage were significantly higher in the HI groups than sham controls at P16 and P29. There were no significant differences between the DHF-PBS, DMSO, and DHF-ARTN Ab groups after HI injury at P16. As compared with the DHF-PBS group, the DMSO and DHF-ARTN Ab groups had increased retinal damage grades at $P 29 . * P<0.05, * \star P<0.01, * \star \star P<0.001$. 
a
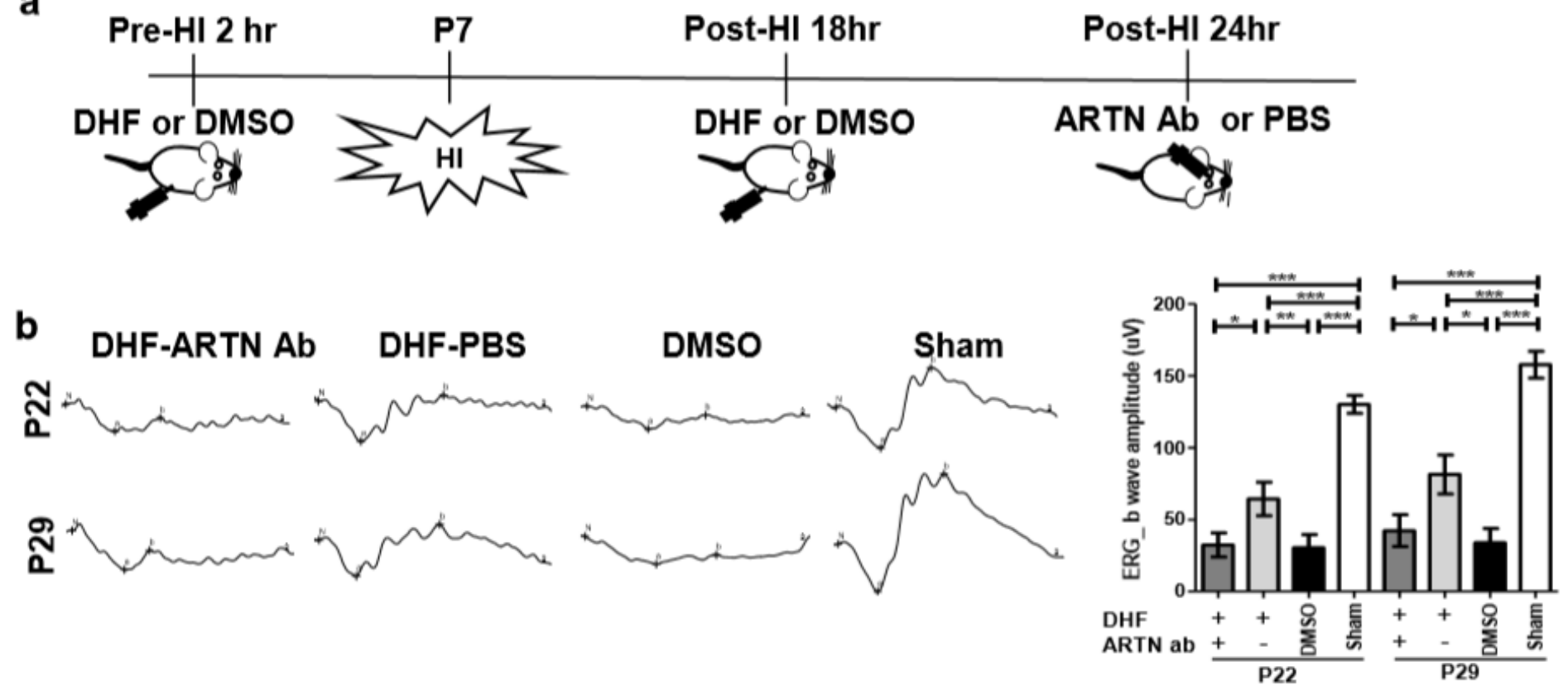

C
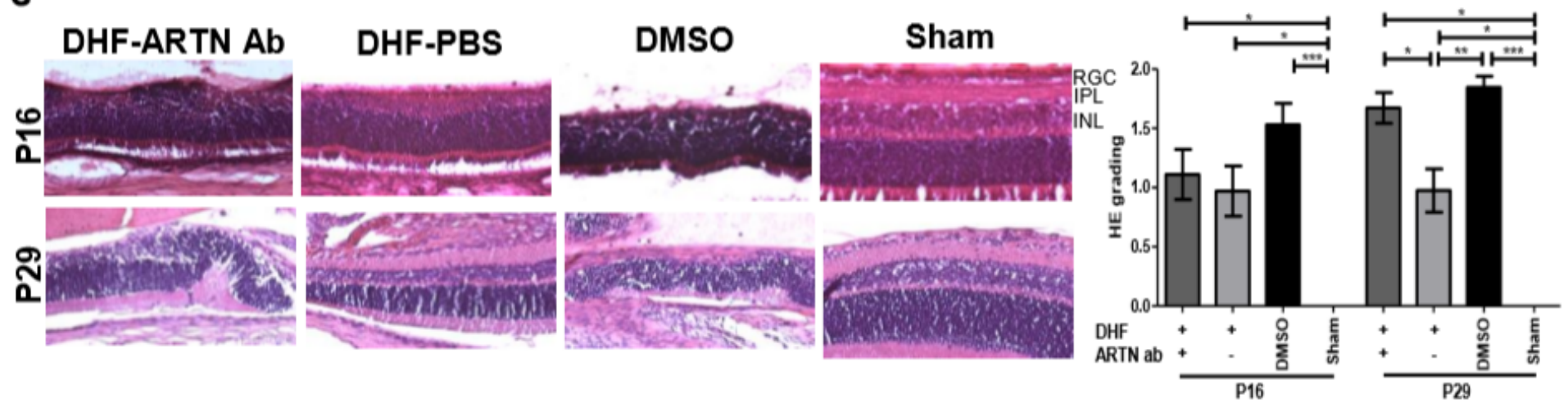

Figure 2

Intravitreal injection of ARTN Antibody inhibits the functional and histological protection of systemic DHF in the HI-injured retinas. (a) Two hours (hrs) before and $18 \mathrm{hrs}$ after the HI, either DHF or DMSO was injected peritoneally. ARTN- Antibody (Ab) or PBS was injected intravitreally at post-HI $24 \mathrm{hrs}$. (b) The retinal function evaluated by ERG demonstrated markedly depressed b-wave amplitudes in the $\mathrm{HI}$ injured groups at P22 and P29 as compared with sham controls. The group data showed the b-wave amplitude in the DHF-PBS group was significantly higher than the DHF-ARTN Ab and DMSO-treated groups after HI at P22 and P29. (c) Representative retinal histologic sections showed the number of RGCs and the thickness of IPL and INL decreased after HI at P16 and P29. The group data showed the grades of retinal damage were significantly higher in the HI groups than sham controls at P16 and P29. There were no significant differences between the DHF-PBS, DMSO, and DHF-ARTN Ab groups after HI injury at P16. As compared with the DHF-PBS group, the DMSO and DHF-ARTN Ab groups had increased retinal damage grades at $P 29 . * P<0.05, * \star P<0.01, * \star \star P<0.001$. 


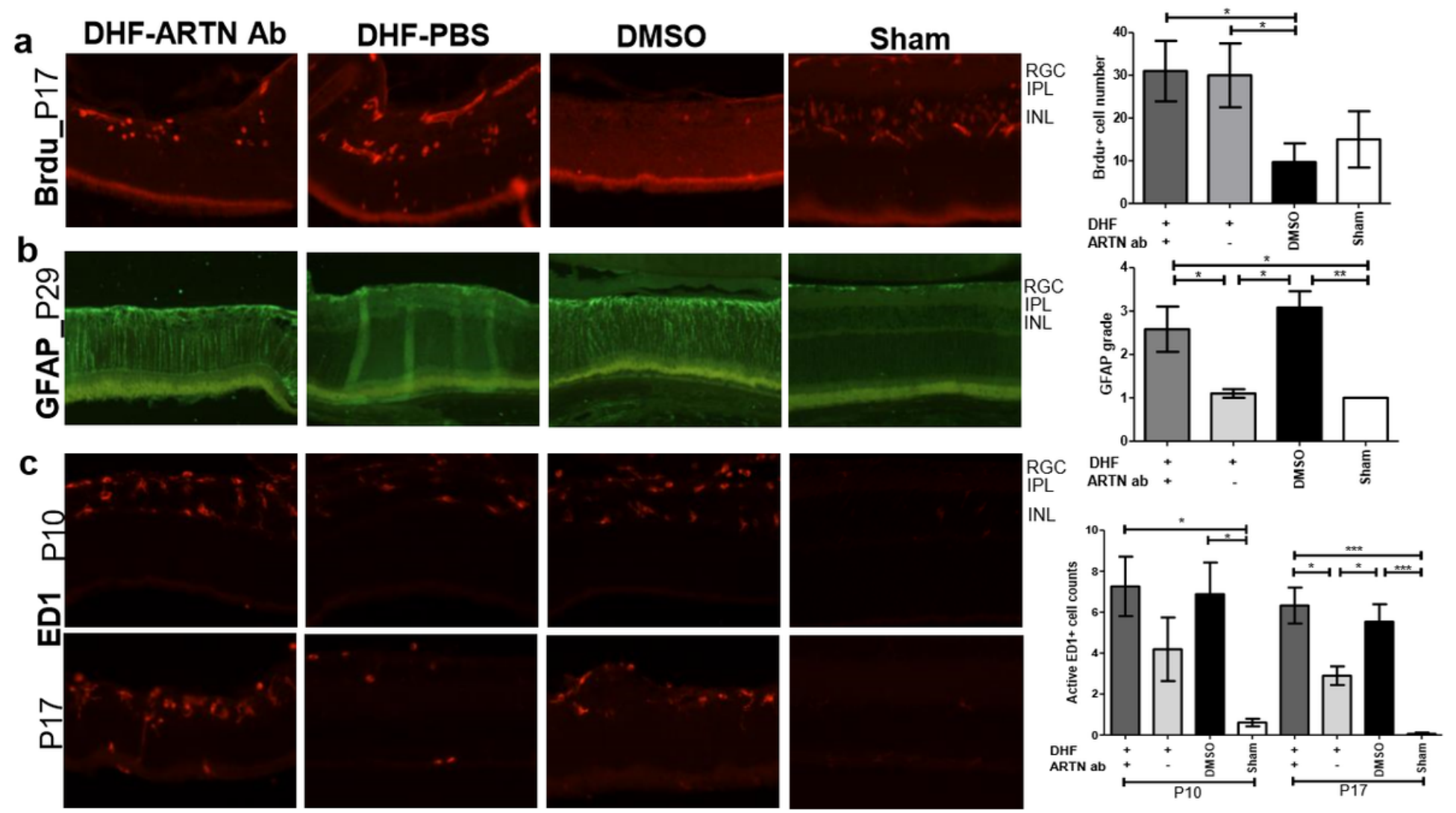

Figure 3

ARTN Antibody blocks the protective effect of systemic DHF in HI-injured retinas by increasing neuroinflammation. (a) At P17, most of Brdu+ cells localized in the RGCs, IPL, and INL. The number of Brdu+ cells was significantly lower in the DMSO-treated group than the DHF-PBS and DHF- ARTN Ab groups after HI. (b) At P29, GFAP immunostaining was quite strong and extensive throughout all retinal layers in the DMSO-treated and DHF-ARTN Ab groups after HI. The grads of GFAP immunoreactivity was significantly higher in the DMSO-treated and DHF-ARTN Ab groups than the DHF-PBS or sham control groups. (c) The prominent ameboid microglial cells (active ED1+ cells) localized in the RGC, IPL, and INL after $\mathrm{HI}$ at P10 and P17. Group data showed the active ED1+ cell counts were markedly increased in the DMSO-treated and DHF-ARTN Ab groups as compared with the sham controls at P10 and P17. After HI, the DHF-PBS group had significantly decreased active ED1+ cells than the DMSO-treated and DHF-ARTN Ab groups at $\mathrm{P} 17 .{ }^{*} \mathrm{P}<0.05,{ }^{*} \mathrm{P}<0.01$, ${ }^{\star * *} \mathrm{P}<0.001$. 


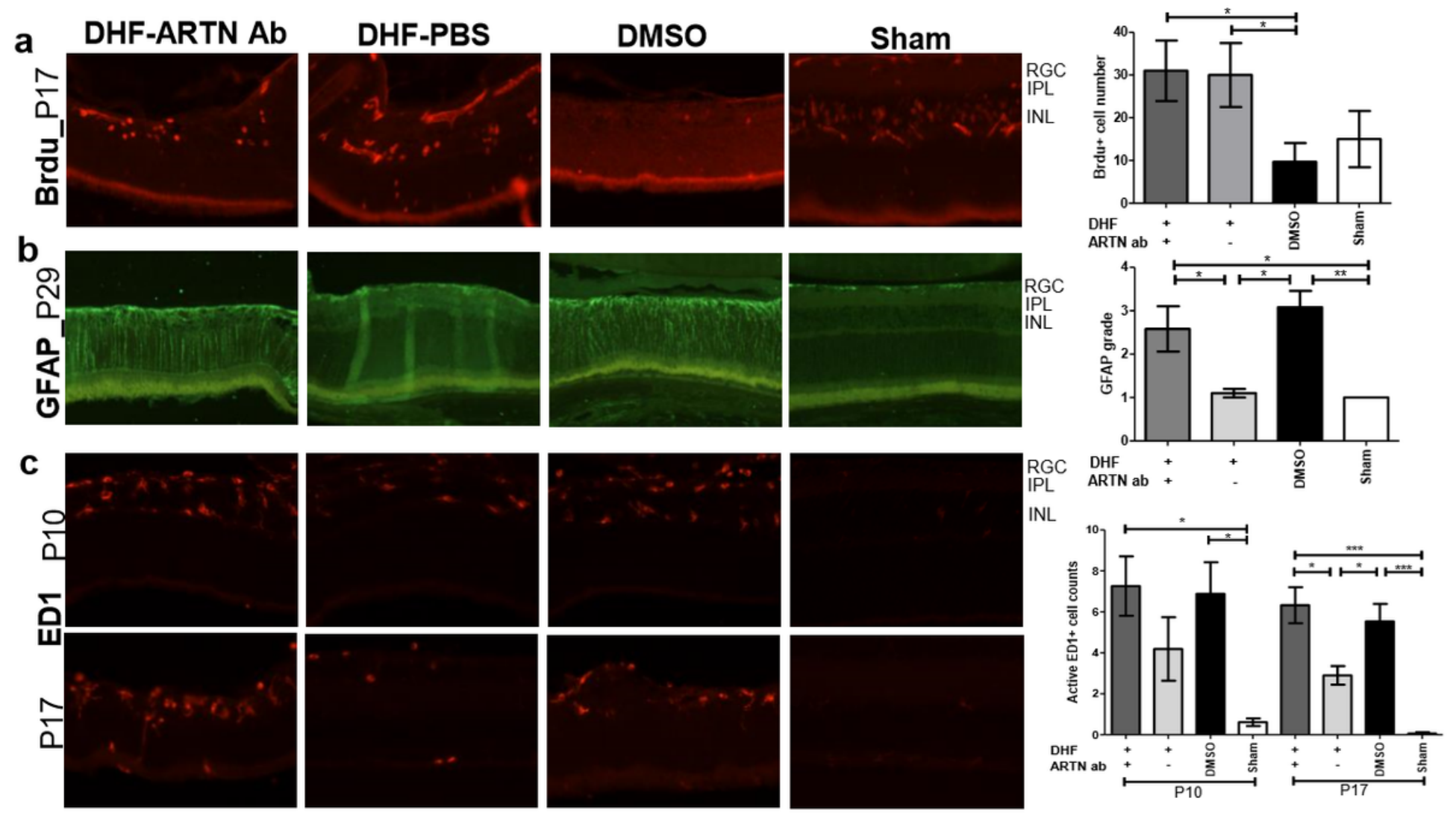

Figure 3

ARTN Antibody blocks the protective effect of systemic DHF in HI-injured retinas by increasing neuroinflammation. (a) At P17, most of Brdu+ cells localized in the RGCs, IPL, and INL. The number of Brdu+ cells was significantly lower in the DMSO-treated group than the DHF-PBS and DHF- ARTN Ab groups after HI. (b) At P29, GFAP immunostaining was quite strong and extensive throughout all retinal layers in the DMSO-treated and DHF-ARTN Ab groups after HI. The grads of GFAP immunoreactivity was significantly higher in the DMSO-treated and DHF-ARTN Ab groups than the DHF-PBS or sham control groups. (c) The prominent ameboid microglial cells (active ED1+ cells) localized in the RGC, IPL, and INL after $\mathrm{HI}$ at P10 and P17. Group data showed the active ED1+ cell counts were markedly increased in the DMSO-treated and DHF-ARTN Ab groups as compared with the sham controls at P10 and P17. After HI, the DHF-PBS group had significantly decreased active ED1+ cells than the DMSO-treated and DHF-ARTN Ab groups at $\mathrm{P} 17 .{ }^{*} \mathrm{P}<0.05,{ }^{*} \mathrm{P}<0.01,{ }^{* *} \mathrm{P}<0.001$. 


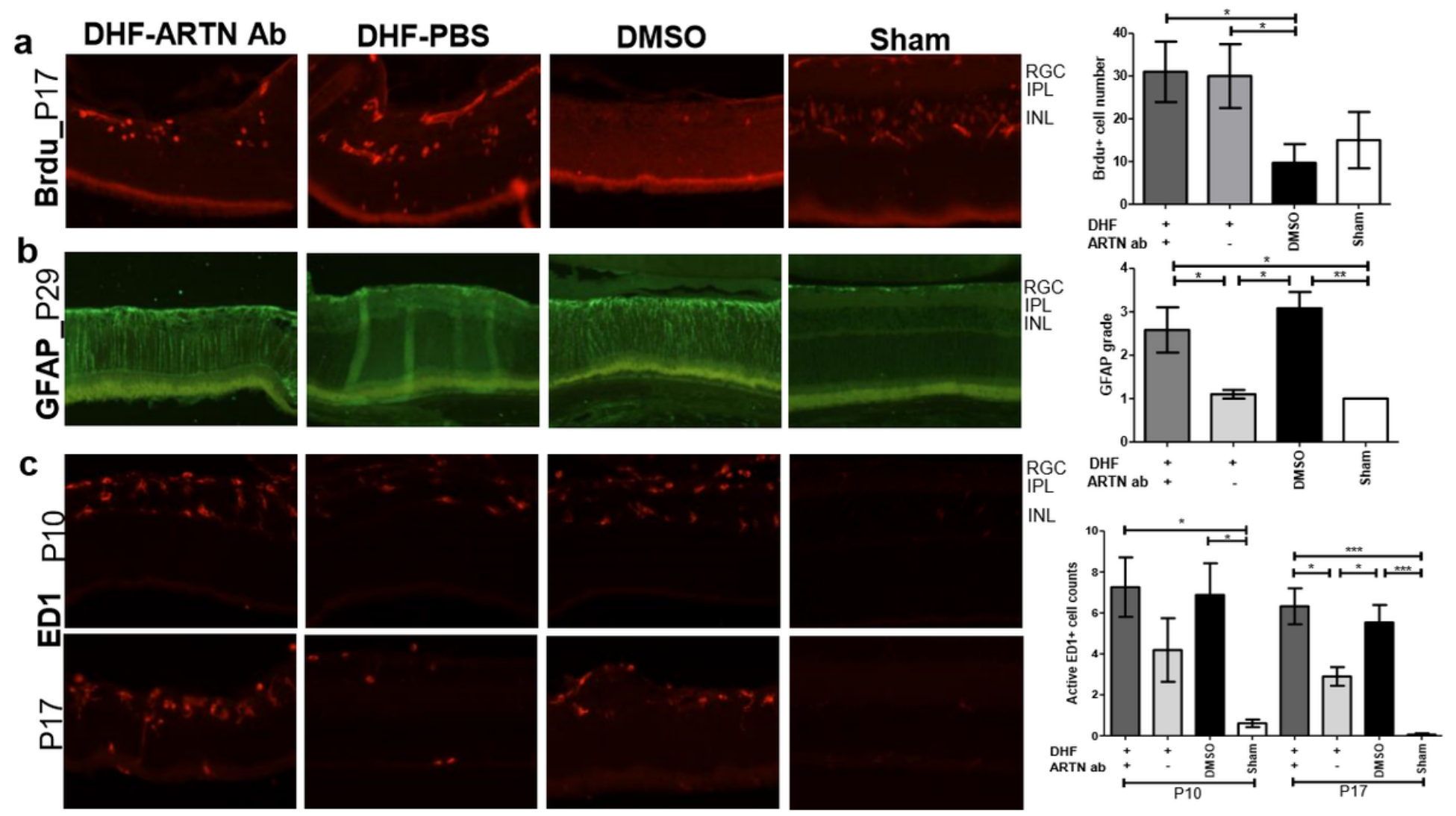

Figure 3

ARTN Antibody blocks the protective effect of systemic DHF in HI-injured retinas by increasing neuroinflammation. (a) At P17, most of Brdu+ cells localized in the RGCs, IPL, and INL. The number of Brdu+ cells was significantly lower in the DMSO-treated group than the DHF-PBS and DHF- ARTN Ab groups after HI. (b) At P29, GFAP immunostaining was quite strong and extensive throughout all retinal layers in the DMSO-treated and DHF-ARTN Ab groups after HI. The grads of GFAP immunoreactivity was significantly higher in the DMSO-treated and DHF-ARTN Ab groups than the DHF-PBS or sham control groups. (c) The prominent ameboid microglial cells (active ED1+ cells) localized in the RGC, IPL, and INL after $\mathrm{HI}$ at P10 and P17. Group data showed the active ED1+ cell counts were markedly increased in the DMSO-treated and DHF-ARTN Ab groups as compared with the sham controls at P10 and P17. After HI, the DHF-PBS group had significantly decreased active ED1+ cells than the DMSO-treated and DHF-ARTN Ab groups at $\mathrm{P} 17 .{ }^{*} \mathrm{P}<0.05,{ }^{*} \mathrm{P}<0.01$, ${ }^{\star * *} \mathrm{P}<0.001$. 
a

\section{ARTN}

ลั

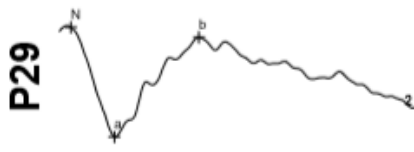
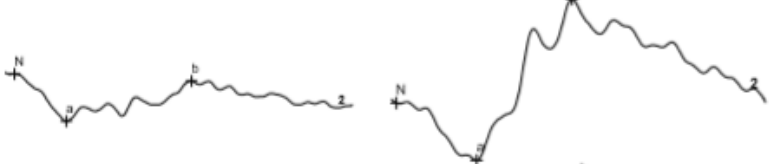

b
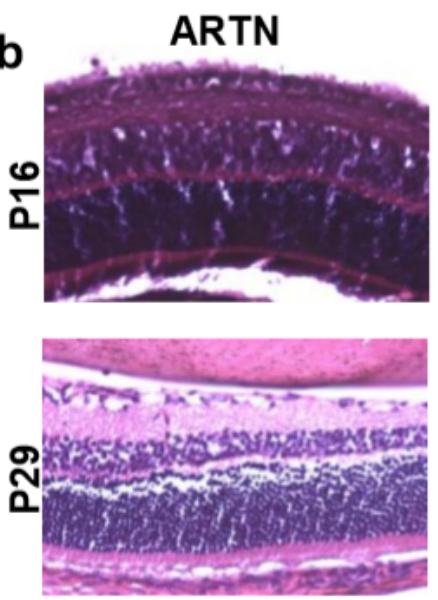
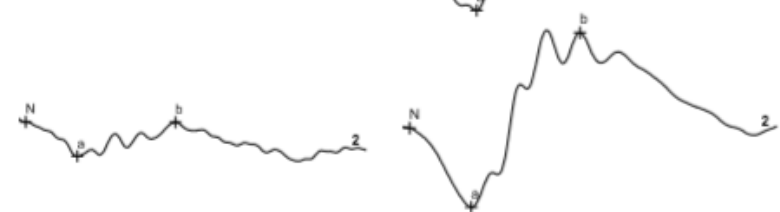

Sham
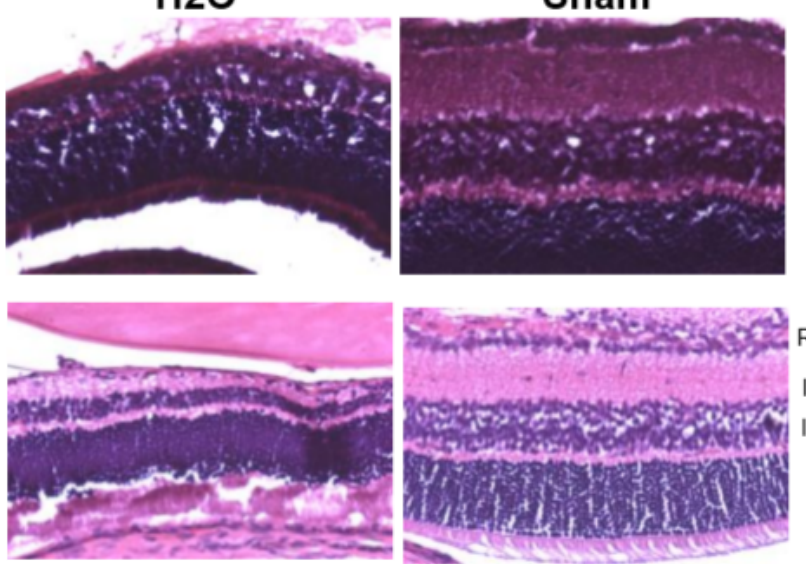
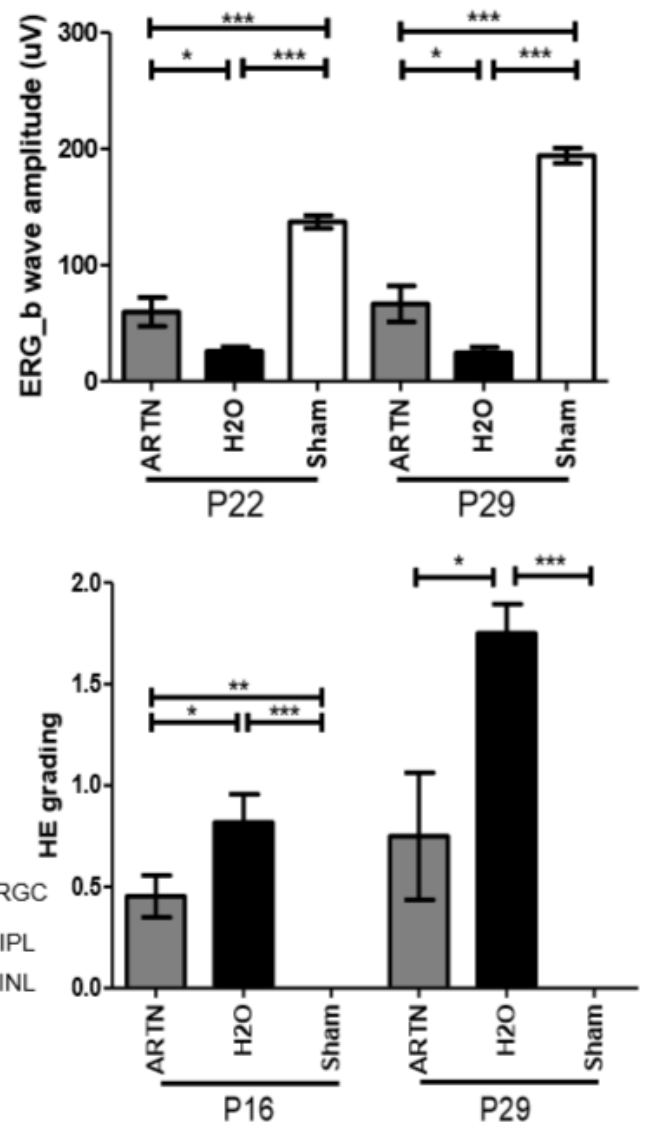

Figure 4

Intravitreal injection of ARTN protects immature retinas against $\mathrm{HI}$ injury in both functional and histological levels. (a) At P22 and P29, the b-wave amplitudes of ERG were significantly decreased in the $\mathrm{HI}$ injured groups, as compared with sham controls. The b-wave amplitude was significantly higher in the ARTN-treated than the H2O-treated group after $\mathrm{HI}$. (b) Representative retinal histologic sections showed the number of RGCs and the thickness of IPL and INL decreased after HI. The group data showed the retinal damage grades were markedly higher in the $\mathrm{H} 2 \mathrm{O}$-treated $\mathrm{HI}$ group than the ARTN-treated $\mathrm{HI}$ group at $\mathrm{P} 16$ and $\mathrm{P} 29$. ${ }^{\star} \mathrm{P}<0.05$, $* * \mathrm{P}<0.01, * \star * \mathrm{P}<0.001$. 
a

ARTN

$\mathrm{H} 2 \mathrm{O}$

ลั

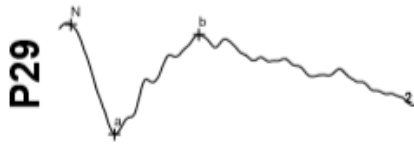
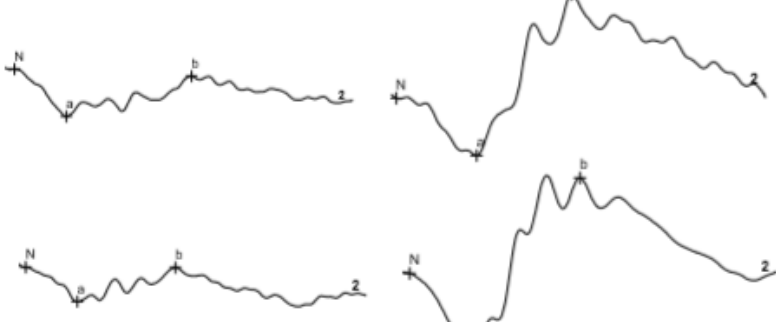

b
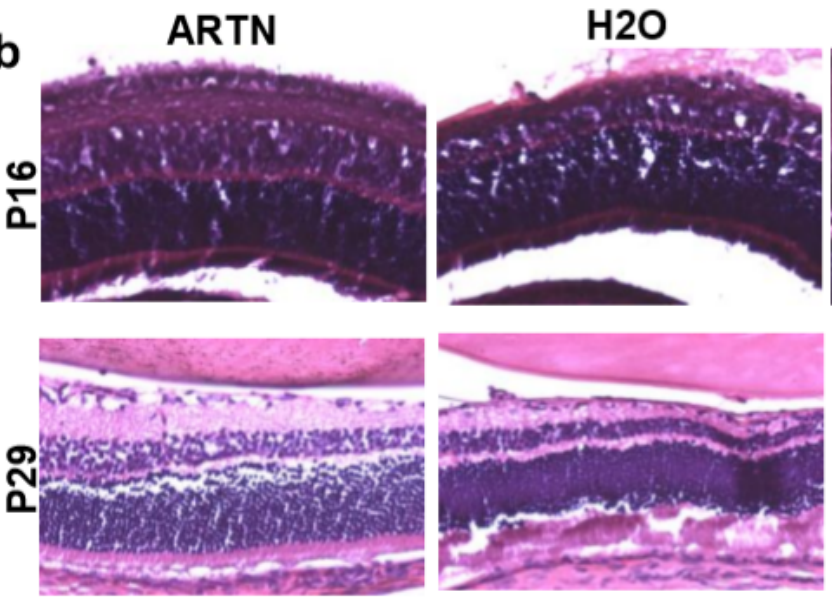

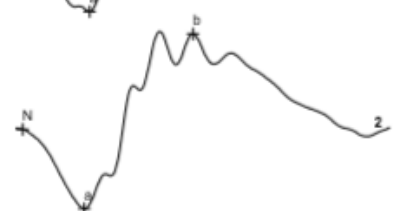

Sham
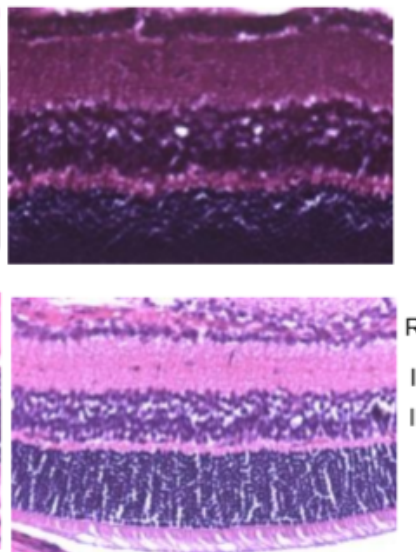
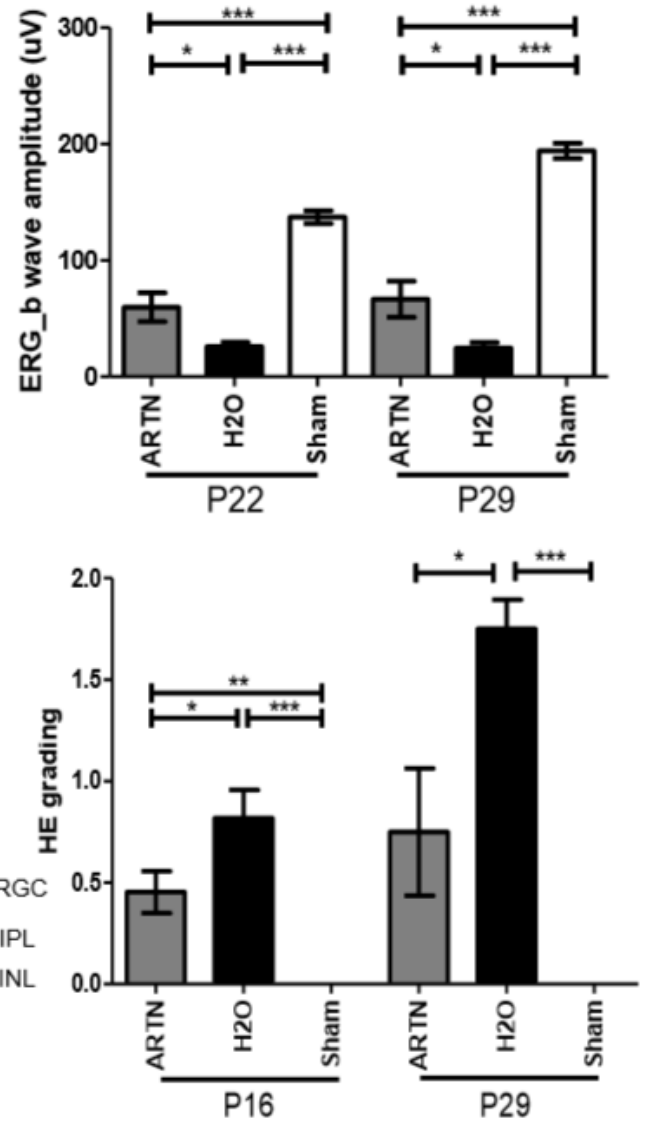

Figure 4

Intravitreal injection of ARTN protects immature retinas against $\mathrm{HI}$ injury in both functional and histological levels. (a) At P22 and P29, the b-wave amplitudes of ERG were significantly decreased in the $\mathrm{HI}$ injured groups, as compared with sham controls. The b-wave amplitude was significantly higher in the ARTN-treated than the H2O-treated group after $\mathrm{HI}$. (b) Representative retinal histologic sections showed the number of RGCs and the thickness of IPL and INL decreased after HI. The group data showed the retinal damage grades were markedly higher in the $\mathrm{H} 2 \mathrm{O}$-treated $\mathrm{HI}$ group than the ARTN-treated $\mathrm{HI}$ group at $\mathrm{P} 16$ and $\mathrm{P} 29$. ${ }^{*} \mathrm{P}<0.05, * \star \mathrm{P}<0.01$, $* \star * \mathrm{P}<0.001$. 
a

ARTN

$\mathrm{H} 2 \mathrm{O}$

ลั

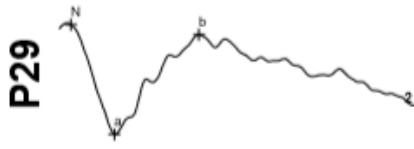
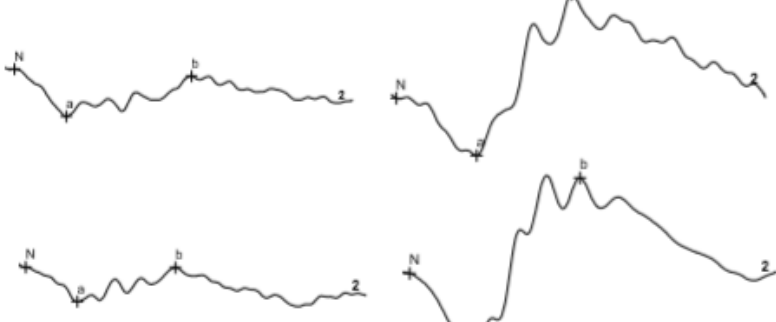

b
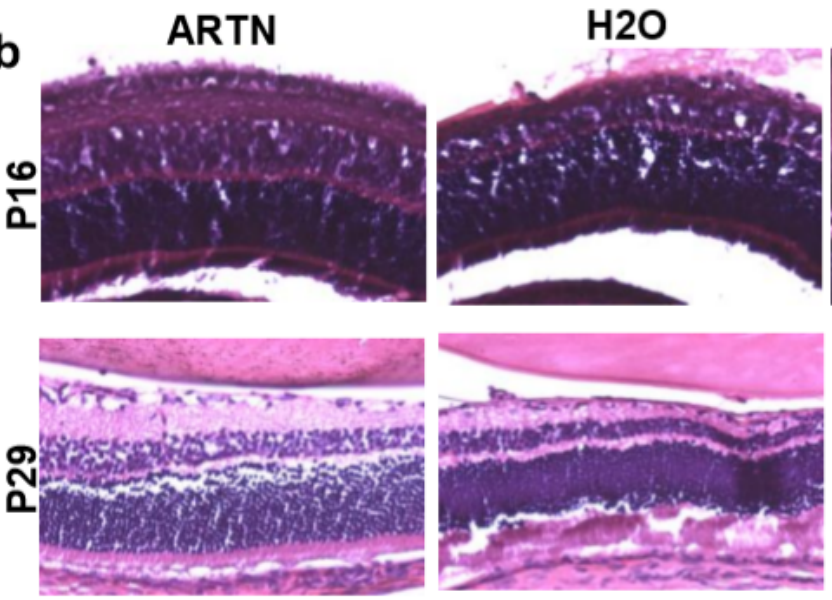

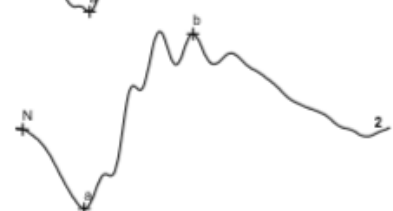

Sham
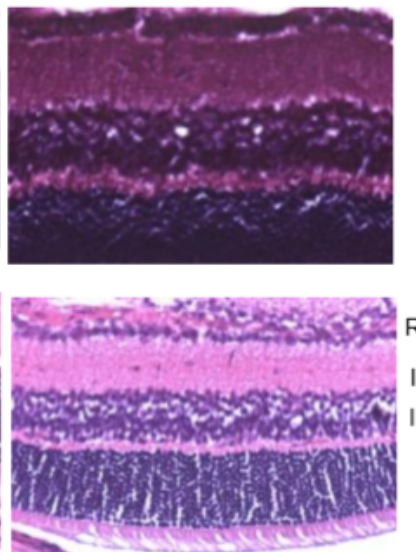
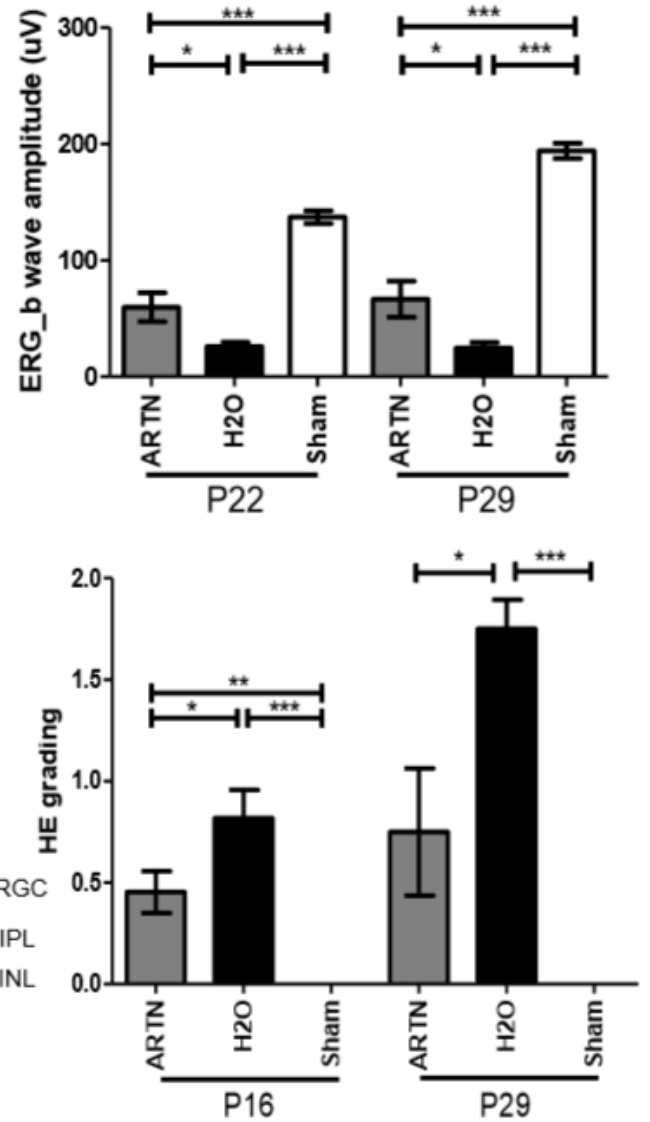

Figure 4

Intravitreal injection of ARTN protects immature retinas against $\mathrm{HI}$ injury in both functional and histological levels. (a) At P22 and P29, the b-wave amplitudes of ERG were significantly decreased in the $\mathrm{HI}$ injured groups, as compared with sham controls. The b-wave amplitude was significantly higher in the ARTN-treated than the H2O-treated group after $\mathrm{HI}$. (b) Representative retinal histologic sections showed the number of RGCs and the thickness of IPL and INL decreased after HI. The group data showed the retinal damage grades were markedly higher in the $\mathrm{H} 2 \mathrm{O}$-treated $\mathrm{HI}$ group than the ARTN-treated $\mathrm{HI}$ group at $\mathrm{P} 16$ and $\mathrm{P} 29$. ${ }^{*} \mathrm{P}<0.05, * \star \mathrm{P}<0.01$, $* \star * \mathrm{P}<0.001$. 

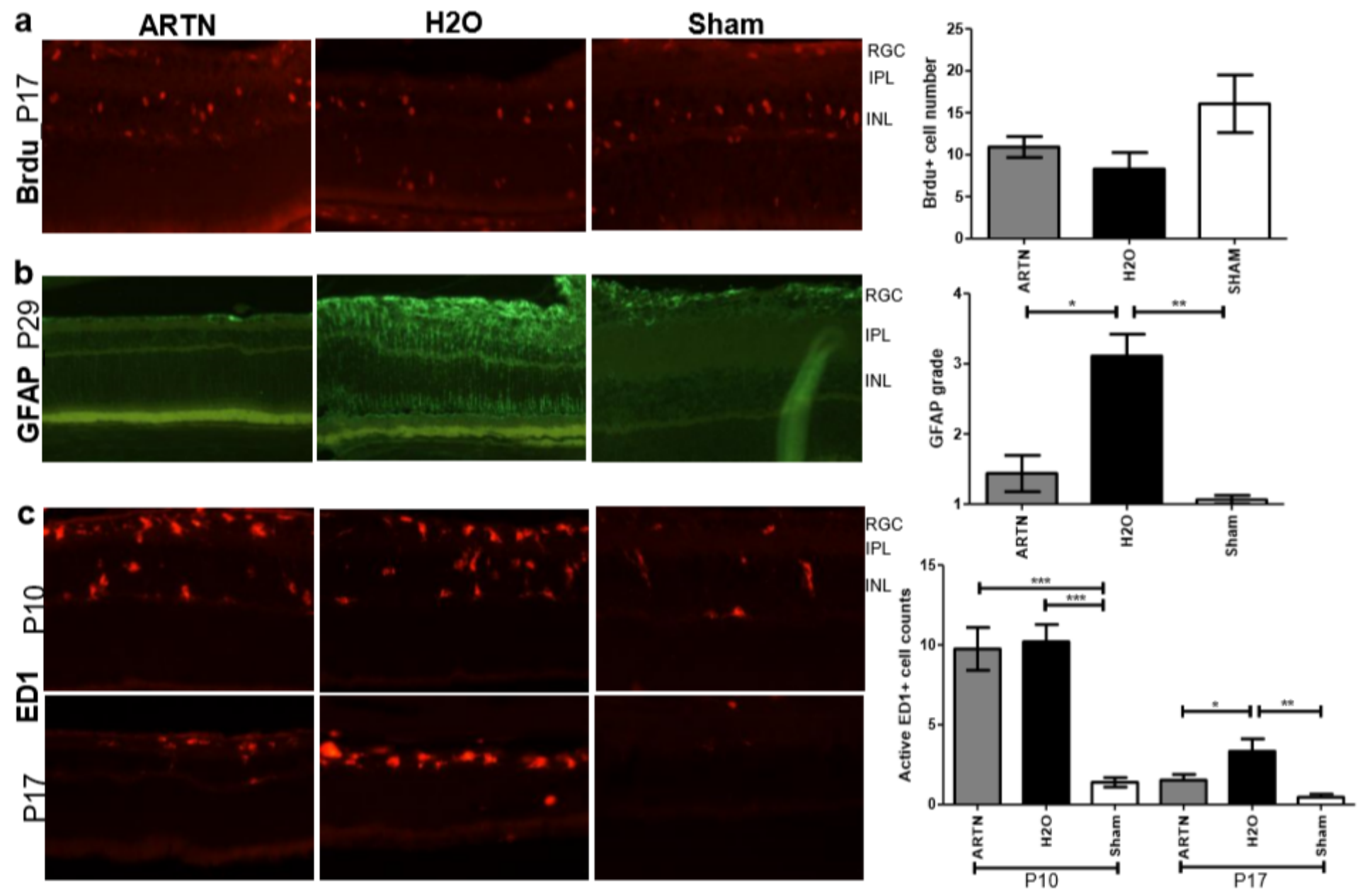

Figure 5

Intravitreal injection of ARTN does not increase cell proliferation but decreases neuroinflammation and astrogliosis. (a) Most of Brdu+ cells are localized in the RGC and INL. At P17, the Brdu+ cell counts were not significantly different between the ARTN- and H2O-treated HI groups. (b) At P29, the GFAP immunoreactivity grades were significantly lower in the ARTN-treated group than the H2O-treated group. (c) Active ED1+ cells localized in the IPL and INL after HI at P10 and P17. The active ED1+ cell counts were significantly increased in the ARTIN-treated and $\mathrm{H} 2 \mathrm{O}$-treated $\mathrm{HI}$ groups as compared with sham controls at P10. The ARTN-treated HI group had markedly decreased active ED1+ cells, as compared with the H2O-treated $\mathrm{HI}$ group at $\mathrm{P} 17$. ${ }^{*} \mathrm{P}<0.05$, $* * \mathrm{P}<0.01$, ${ }^{\star \star *} \mathrm{P}<0.001$. 

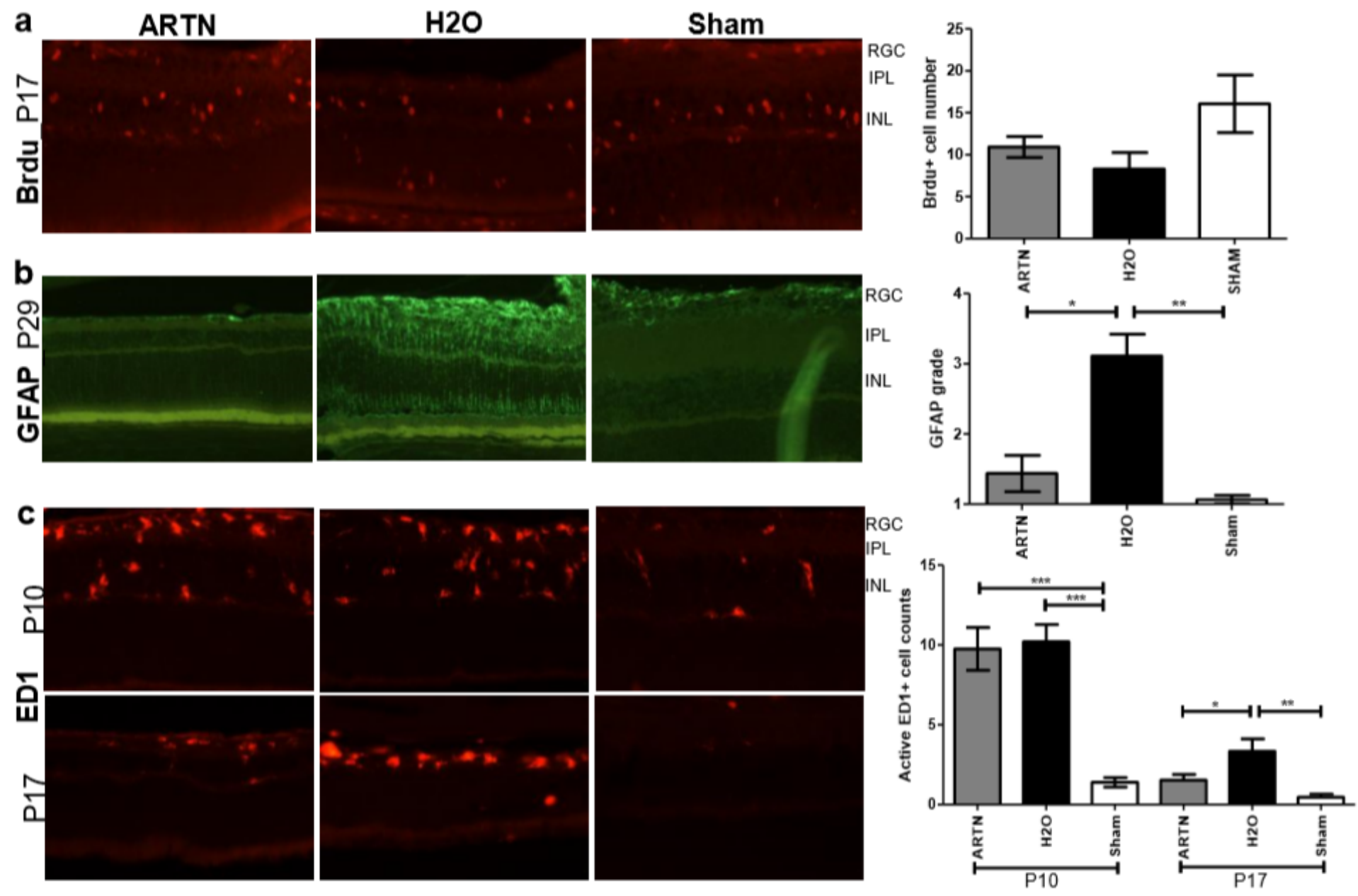

Figure 5

Intravitreal injection of ARTN does not increase cell proliferation but decreases neuroinflammation and astrogliosis. (a) Most of Brdu+ cells are localized in the RGC and INL. At P17, the Brdu+ cell counts were not significantly different between the ARTN- and H2O-treated HI groups. (b) At P29, the GFAP immunoreactivity grades were significantly lower in the ARTN-treated group than the H2O-treated group. (c) Active ED1+ cells localized in the IPL and INL after HI at P10 and P17. The active ED1+ cell counts were significantly increased in the ARTIN-treated and $\mathrm{H} 2 \mathrm{O}$-treated $\mathrm{HI}$ groups as compared with sham controls at P10. The ARTN-treated HI group had markedly decreased active ED1+ cells, as compared with the H2O-treated $\mathrm{HI}$ group at $\mathrm{P} 17$. ${ }^{*} \mathrm{P}<0.05$, $* * \mathrm{P}<0.01$, ${ }^{\star \star *} \mathrm{P}<0.001$. 

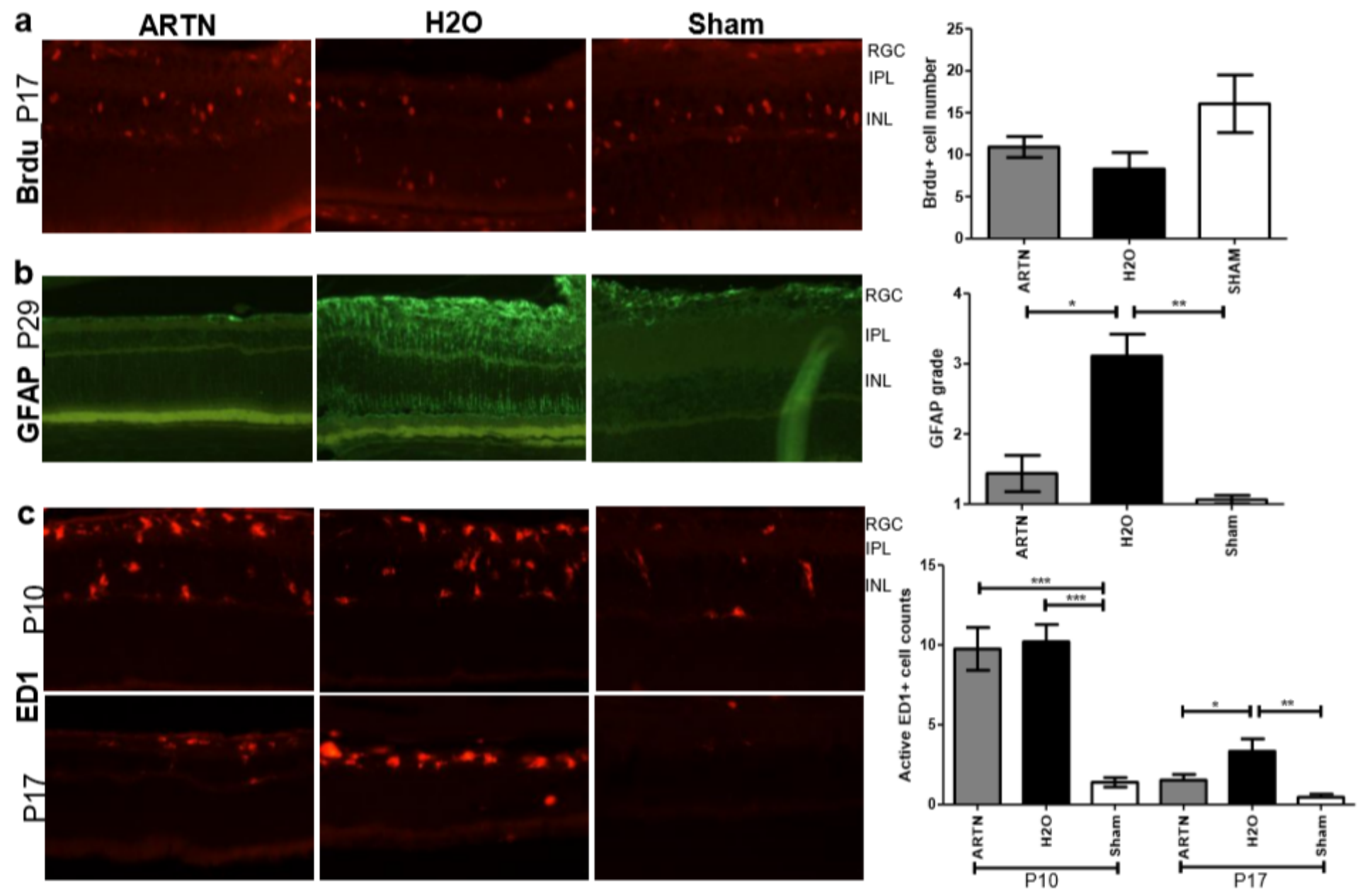

Figure 5

Intravitreal injection of ARTN does not increase cell proliferation but decreases neuroinflammation and astrogliosis. (a) Most of Brdu+ cells are localized in the RGC and INL. At P17, the Brdu+ cell counts were not significantly different between the ARTN- and H2O-treated HI groups. (b) At P29, the GFAP immunoreactivity grades were significantly lower in the ARTN-treated group than the H2O-treated group. (c) Active ED1+ cells localized in the IPL and INL after HI at P10 and P17. The active ED1+ cell counts were significantly increased in the ARTIN-treated and $\mathrm{H} 2 \mathrm{O}$-treated $\mathrm{HI}$ groups as compared with sham controls at P10. The ARTN-treated HI group had markedly decreased active ED1+ cells, as compared with the H2O-treated $\mathrm{HI}$ group at $\mathrm{P} 17$. ${ }^{*} \mathrm{P}<0.05$, $* * \mathrm{P}<0.01$, ${ }^{\star \star *} \mathrm{P}<0.001$. 

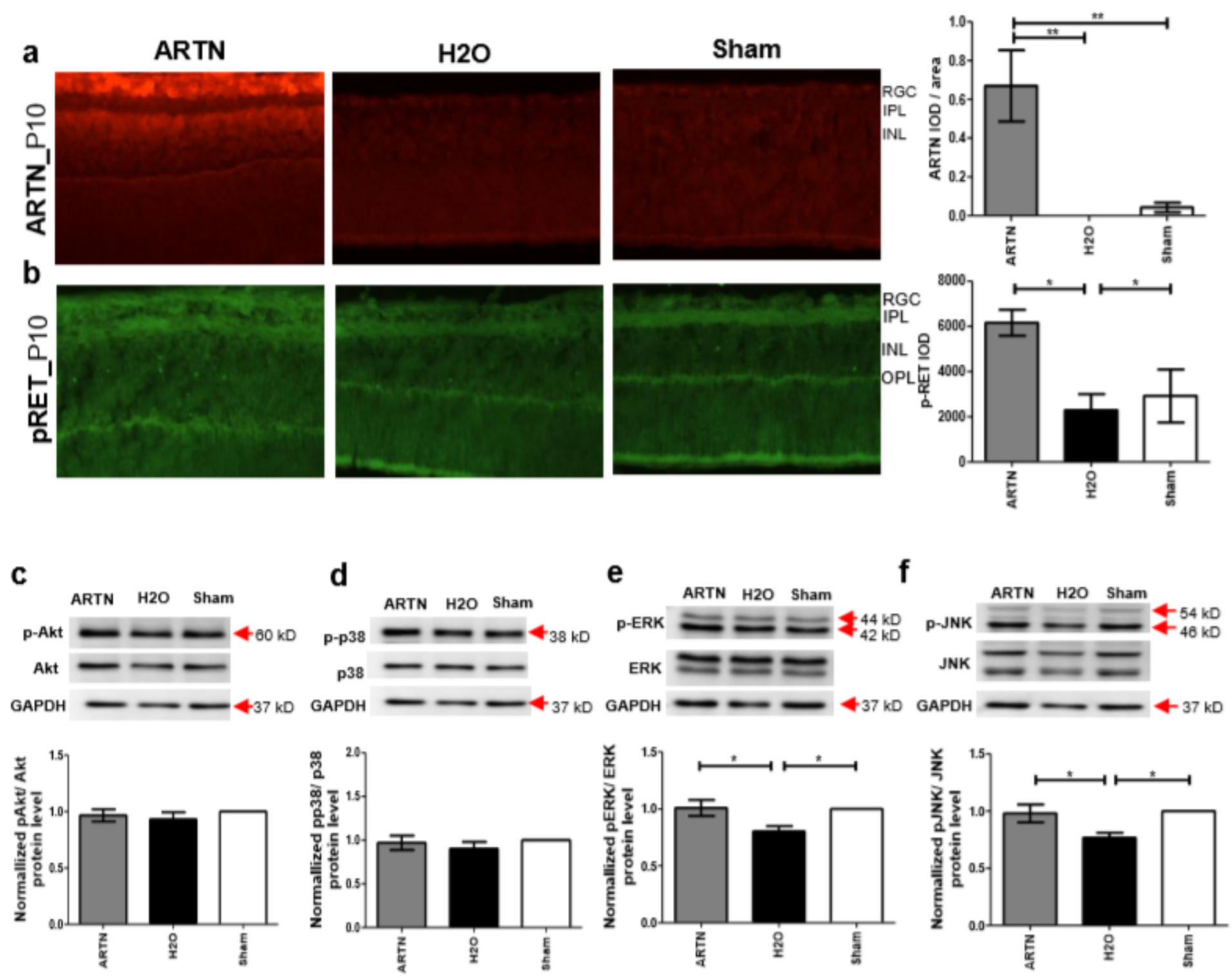

\section{Figure 6}

Post-treatment with ARTN enhances RET, ERK, and JNK phosphorylation in the immature retina after HI injury. (a) At P10, the ARTN immunostaining was prominent in the RGC and INL of the ARTN-treated HI group. Group data showed the immunoreactivity of ARTN was significantly higher in the ARTN-treated HI group than the H2O-treated $\mathrm{HI}$ group and sham controls. (b) Alt P10, the immunostaining of phosphorylated RET (pRET) localized in the RGC, IPL, and OPL. Group data showed the H2O-treated HI group had significantly decreased pRET immunostaining as compared with the ARTN-treated HI groups and sham controls. At P10, western blot analysis showed there were no significant differences in the (c) phosphorylated (p) Akt or (d) pp38 between the ARTN-treated HI group, H2O-treated HI group, and sham controls. The ARTN-treated HI group had a higher expression of (e) pERK and (f) pJNK than the H2Otreated HI group. ${ }^{*} \mathrm{P}<0.05$, ** $\mathrm{P}<0.01$, $* \star * P<0.001$. 

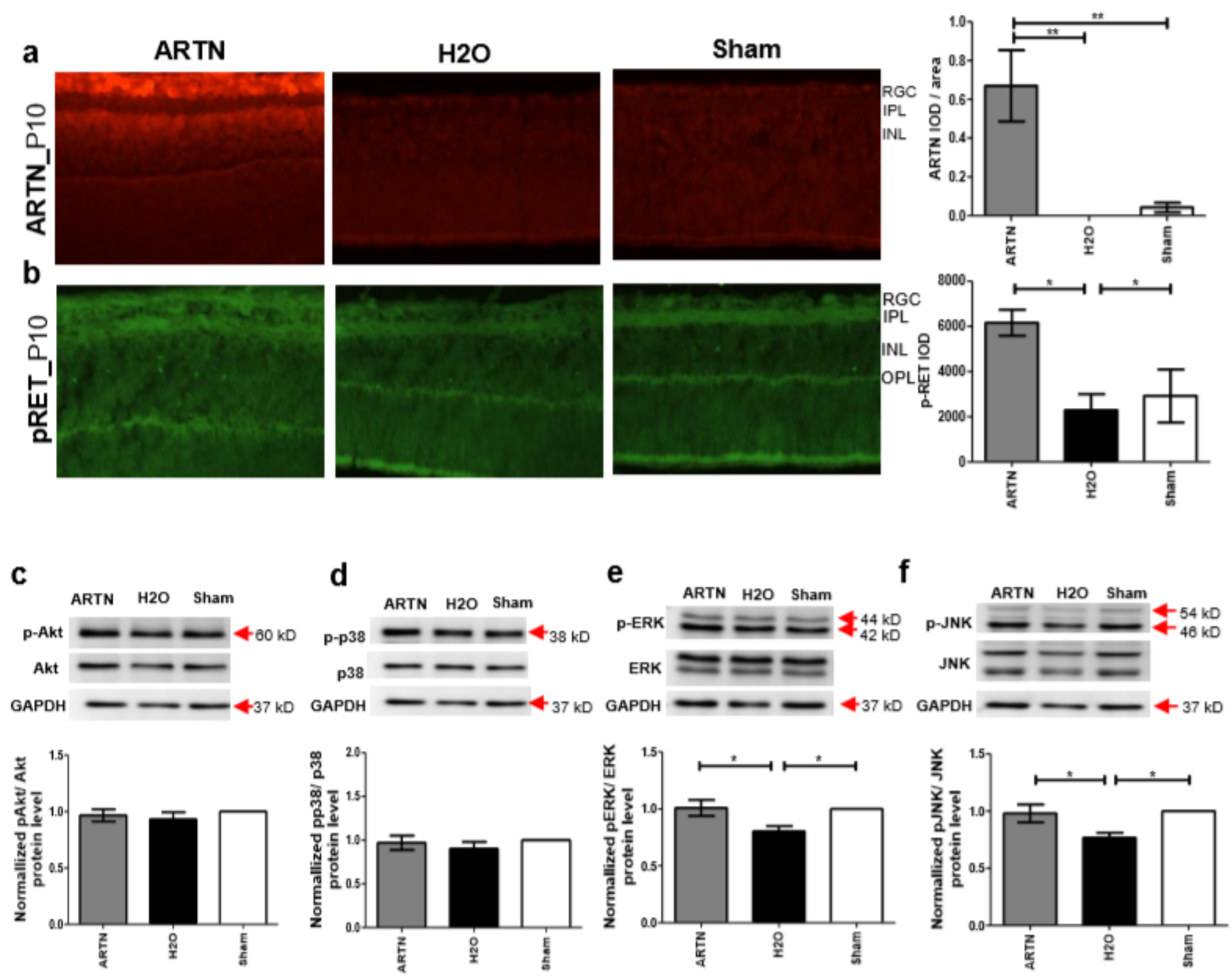

\section{Figure 6}

Post-treatment with ARTN enhances RET, ERK, and JNK phosphorylation in the immature retina after HI injury. (a) At P10, the ARTN immunostaining was prominent in the RGC and INL of the ARTN-treated HI group. Group data showed the immunoreactivity of ARTN was significantly higher in the ARTN-treated HI group than the H2O-treated $\mathrm{HI}$ group and sham controls. (b) Alt P10, the immunostaining of phosphorylated RET (pRET) localized in the RGC, IPL, and OPL. Group data showed the H2O-treated HI group had significantly decreased pRET immunostaining as compared with the ARTN-treated HI groups and sham controls. At P10, western blot analysis showed there were no significant differences in the (c) phosphorylated (p) Akt or (d) pp38 between the ARTN-treated HI group, H2O-treated HI group, and sham controls. The ARTN-treated HI group had a higher expression of (e) pERK and (f) pJNK than the H2Otreated HI group. ${ }^{*} \mathrm{P}<0.05$, ** $\mathrm{P}<0.01$, $* \star * P<0.001$. 

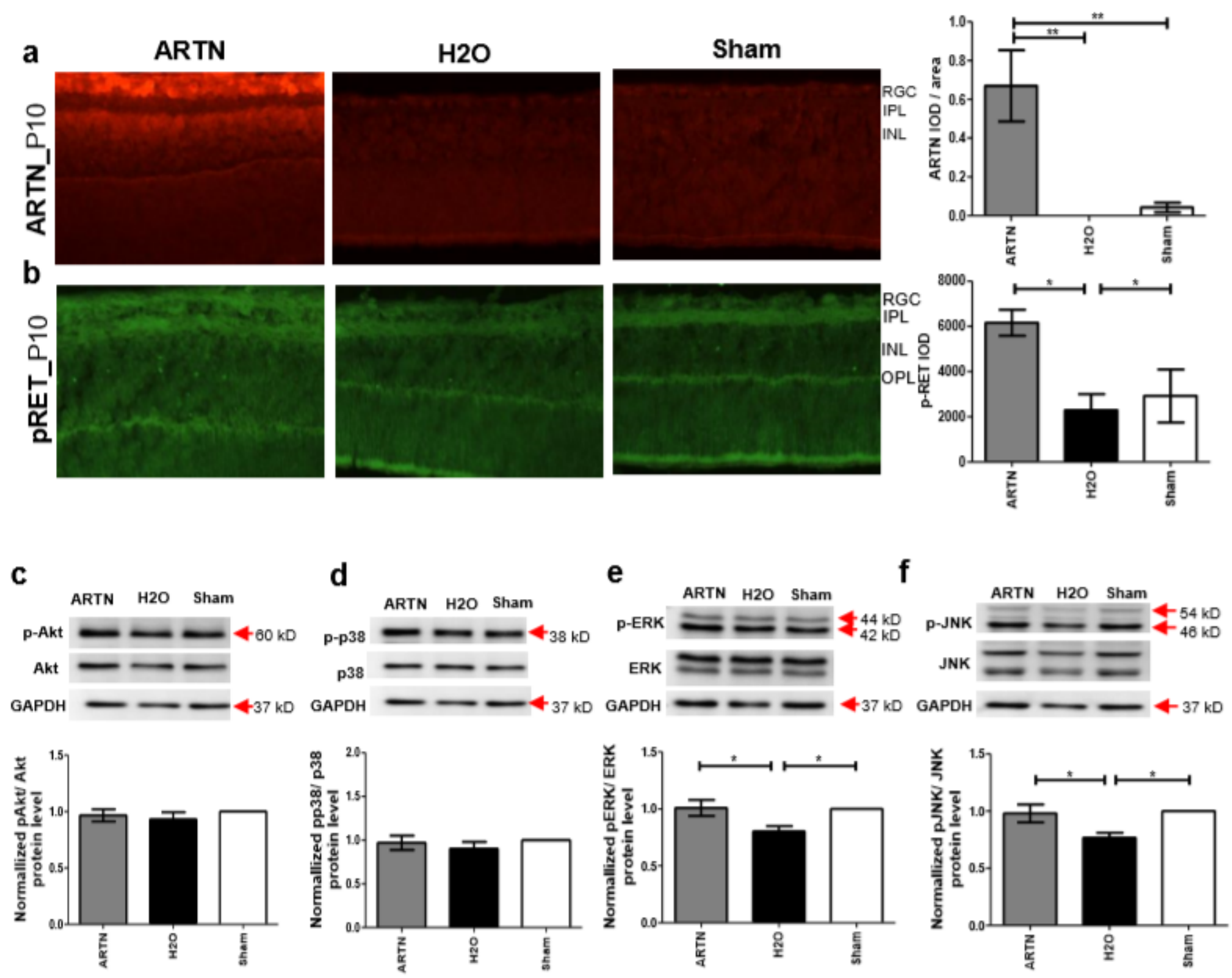

\section{Figure 6}

Post-treatment with ARTN enhances RET, ERK, and JNK phosphorylation in the immature retina after HI injury. (a) At P10, the ARTN immunostaining was prominent in the RGC and INL of the ARTN-treated HI group. Group data showed the immunoreactivity of ARTN was significantly higher in the ARTN-treated HI group than the H2O-treated $\mathrm{HI}$ group and sham controls. (b) Alt P10, the immunostaining of phosphorylated RET (pRET) localized in the RGC, IPL, and OPL. Group data showed the H2O-treated HI group had significantly decreased pRET immunostaining as compared with the ARTN-treated HI groups and sham controls. At P10, western blot analysis showed there were no significant differences in the (c) phosphorylated (p) Akt or (d) pp38 between the ARTN-treated HI group, H2O-treated HI group, and sham controls. The ARTN-treated HI group had a higher expression of (e) pERK and (f) pJNK than the H2Otreated HI group. ${ }^{*} \mathrm{P}<0.05$, ** $\mathrm{P}<0.01$, $* \star * P<0.001$. 


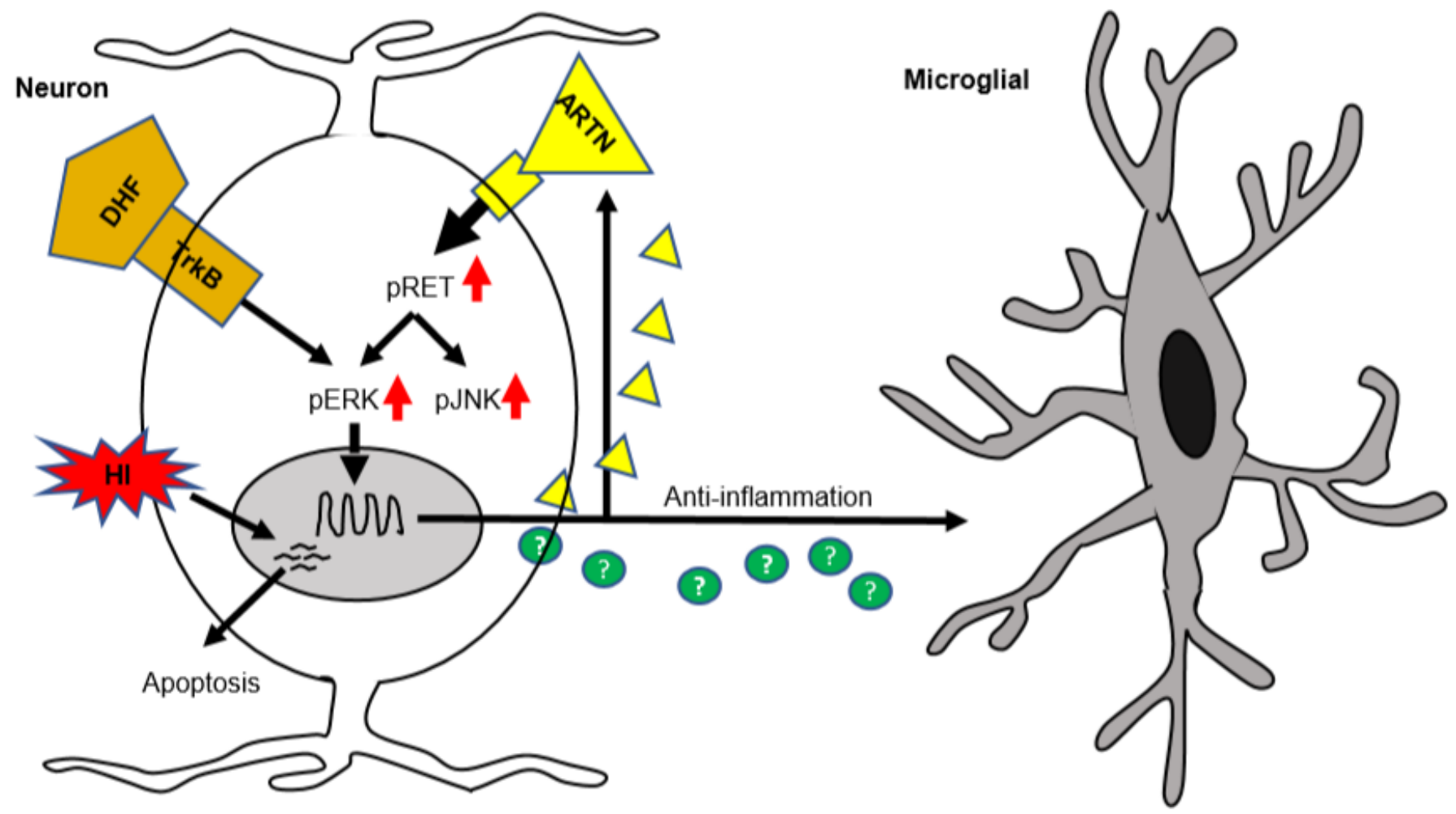

Figure 7

Summary of the role of ARTN in the immature retinas after HI Systemic DHF treatment can activate pERK and increase endogenous ARTN to reduce microglia response and provide long-term neuroprotection. IVI ARTN antibodies block this protective effect. Similarly, the exogenous ARTN provided by IVI can activate pRET, pERK, and pJNK, which can also reduce the activation of microglia and protect the immature retina from $\mathrm{HI}$ damage. All research results show that ARTN has the potential to provide long-term neuroprotection, and its protection may be to reduce $\mathrm{HI}$ damage in immature retinas by reducing retinal inflammation. 


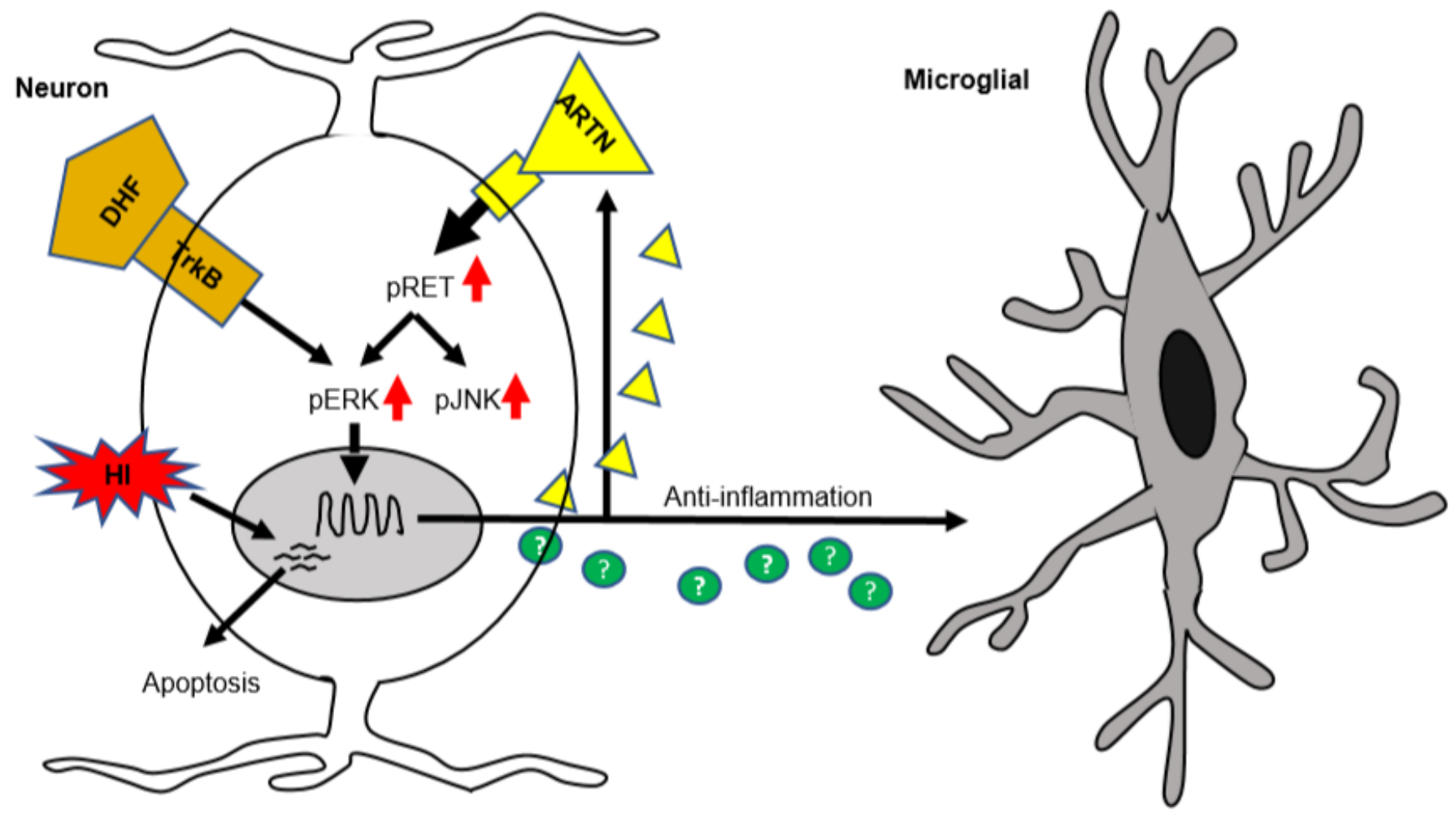

Figure 7

Summary of the role of ARTN in the immature retinas after HI Systemic DHF treatment can activate pERK and increase endogenous ARTN to reduce microglia response and provide long-term neuroprotection. IVI ARTN antibodies block this protective effect. Similarly, the exogenous ARTN provided by IVI can activate pRET, pERK, and pJNK, which can also reduce the activation of microglia and protect the immature retina from $\mathrm{HI}$ damage. All research results show that ARTN has the potential to provide long-term neuroprotection, and its protection may be to reduce $\mathrm{HI}$ damage in immature retinas by reducing retinal inflammation. 


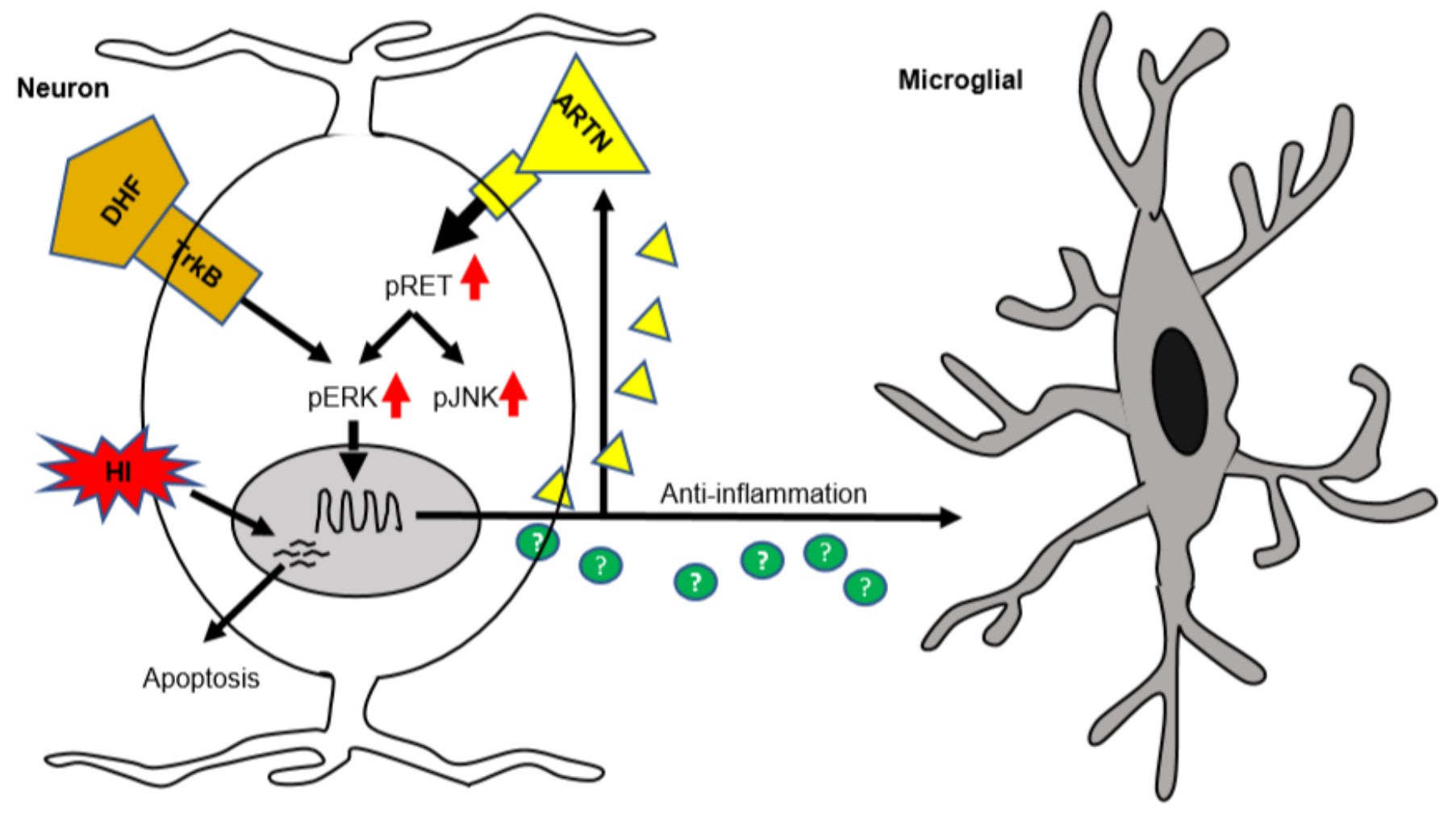

Figure 7

Summary of the role of ARTN in the immature retinas after HI Systemic DHF treatment can activate pERK and increase endogenous ARTN to reduce microglia response and provide long-term neuroprotection. IVI ARTN antibodies block this protective effect. Similarly, the exogenous ARTN provided by IVI can activate pRET, pERK, and pJNK, which can also reduce the activation of microglia and protect the immature retina from $\mathrm{HI}$ damage. All research results show that ARTN has the potential to provide long-term neuroprotection, and its protection may be to reduce $\mathrm{HI}$ damage in immature retinas by reducing retinal inflammation.

\section{Supplementary Files}

This is a list of supplementary files associated with this preprint. Click to download.

- Additionalfile1.pdf

- Additionalfile1.pdf

- Additionalfile1.pdf

- Additionalfile2.pdf

- Additionalfile2.pdf

- Additionalfile2.pdf 
- SupplementaryfilerawimageofWB.pdf

- SupplementaryfilerawimageofWB.pdf

- SupplementaryfilerawimageofWB.pdf 